\title{
Some examples of universal and generic partial orders
}

\author{
Jan Hubička and Jaroslav Nešetřil
}

\begin{abstract}
We survey structures endowed with natural partial orderings and prove their universality. These partial orders include partial orders on sets of words, partial orders formed by geometric objects, grammars, polynomials and homomorphism order for various combinatorial objects.
\end{abstract}

\section{Introduction}

For given class $\mathcal{K}$ of countable partial orders we say that class $\mathcal{K}$ contains an embedding-universal (or simply universal) structure $\left(U, \leq_{U}\right)$ if every partial order $\left(P, \leq_{P}\right) \in \mathcal{K}$ can be found as induced suborder of $\left(U, \leq_{U}\right)$ (or in other words, there exists embedding from $\left(P, \leq_{P}\right)$ to $\left.\left(U, \leq_{U}\right)\right)$.

A partial order $\left(P, \leq_{P}\right)$ is ultrahomogeneous (or simply homogeneous), if every isomorphism of finite suborders of $\left(P, \leq_{P}\right)$ can be extended to an automorphism of $\left(P, \leq_{P}\right)$.

A partial order $\left(P, \leq_{P}\right)$ is generic if it is both ultrahomogeneous and universal.

The generic objects can be obtained from the Fraïssé limit [4]. But it is important that often these generic objects (despite their apparent complexity and universality) admit a concise presentation. Thus for example the Rado graph (i.e. countable universal and homogeneous undirected graph) can be represented in various ways by elementary properties of sets or finite sequences, number theory or even probability. Similar concise representations were found for some other generic objects such as all undirected ultrahomogeneous graphs [9] or the Urysohn space [7]. The study of generic partial order also motivated this paper and we consider representation of the generic partial order in Section 3.

The notion of finite presentation we interpret here broadly as a succinct representation of an infinite set, succint in the sense that elements are finite models with relations induced by "compatible mappings" (such as homomorphisms) between the corresponding models. This intuitive definition suffices as we are interested in the (positive) examples of such representations.

2010 Mathematics Subject Classification. Primary 06A06, 06A11; Secondary 05C38.

Key words and phrases. universal partial order, generic partial order, path homomorphisms, homomorphism order, order of convex sets, words order, Conway numbers.

The Institute for Theoretical Computer Science (ITI) is supported as project 1M0545 by the Ministry of Education of the Czech Republic. 
A finite presentation of the generic partial order is given in $[\mathbf{9}]$ - however this construction is quite complicated. This paper gives a more streamlined construction and relates it to Conway surreal numbers (see Section 3).

In Section 2 we present several simple constructions which yield (countably) universal partial orders. Such objects are interesting on their own and were intensively studied in the context of universal algebra and categories. For example, it is a classical result of Pultr and Trnková [20] that finite graphs with the homomorphism order are countably universal quasiorder. Extending and completing [9] we give here several constructions which yields to universal partial orders. These constructions include:

(1) The order $\left(\mathcal{W}, \leq_{\mathcal{W}}\right)$ on sets of words in the alphabet $\{0,1\}$.

(2) The dominance order on the binary tree $\left(\mathcal{B}, \leq_{\mathcal{B}}\right)$.

(3) The inclusion order of finite sets of finite intervals $\left(\mathcal{I}, \leq_{\mathcal{I}}\right)$.

(4) The inclusion order of convex hulls of finite sets of points in the plane $\left(\mathcal{C}, \leq_{\mathcal{C}}\right)$

(5) The order of piecewise linear functions on rationals $\left(\mathcal{F}, \leq_{\mathcal{F}}\right)$.

(6) The inclusion order of periodic sets $(\mathcal{S}, \subseteq)$.

(7) The order of sets of truncated vectors (generalization of orders of vectors of finite dimension) $(\mathcal{T} V, \leq \mathcal{T} V)$.

(8) The orders implied by grammars on words $\left(\mathcal{G}, \leq_{\mathcal{G}}\right)$.

(9) The homomorphism order of oriented paths $\left(\mathcal{P}, \leq_{\mathcal{P}}\right)$.

Note that with universal partial orders we have more freedom (than with the generic pratial order) and as a consequence we give a perhaps surprising variety of finite presentations.

We start with a simple representation by means of finite sets of binary words. This representation seems to capture properties of such a universal partial order very well and it will serve as our "master" example. In most other cases we prove the universality of some particular partial order by finding a mapping from the words representation into the structure in question. This technique will be shown in several applications in the next sections. While some of these structures are known be universal, see e.g. $[\mathbf{5}, \mathbf{1 6}, \mathbf{8}]$, in several cases we can prove the universality in a new, we believe, much easier way. The embeddings of structures are presented in Figure 1 (ones denoted by dotted lines are not presented in this paper, but references are given).

At this point we would like to mention that the (countable) universality is an essentially finite problem as it can be formulated as follows: By an on-line representation of a class $\mathcal{K}$ of partial orders in a partial order $\left(P, \leq_{P}\right)$, we mean that one can construct an embedding $\varphi: R \rightarrow P$ of any partial order $\left(R, \leq_{R}\right)$ in class $\mathcal{K}$ under the restriction that the elements of $R$ are revealed one by one. The on-line representation of a class of partial orders can be considered as a game between two players $A$ and $B$ (usually Alice and Bob). Player $B$ chooses a partial order $\left(P, \leq_{P}\right)$ in the class $\mathcal{K}$, and reveals the elements of $P$ one by one to player $A$ ( $B$ is a bad guy). Whenever an element of $x$ of $P$ is revealed to $A$, the relations among $x$ and previously revealed elements are also revealed. Player $A$ is required to assign a vertex $\varphi(x)$-before the next element is revealed - such that $\varphi$ is an embedding of the suborder induced by $\left(P, \leq_{P}\right)$ on the already revealed elements of $\left(R, \leq_{R}\right)$. Player $A$ wins the game if he succeeds in constructing an embedding $\varphi$. 
Order implied by clones on boolean functions, 5

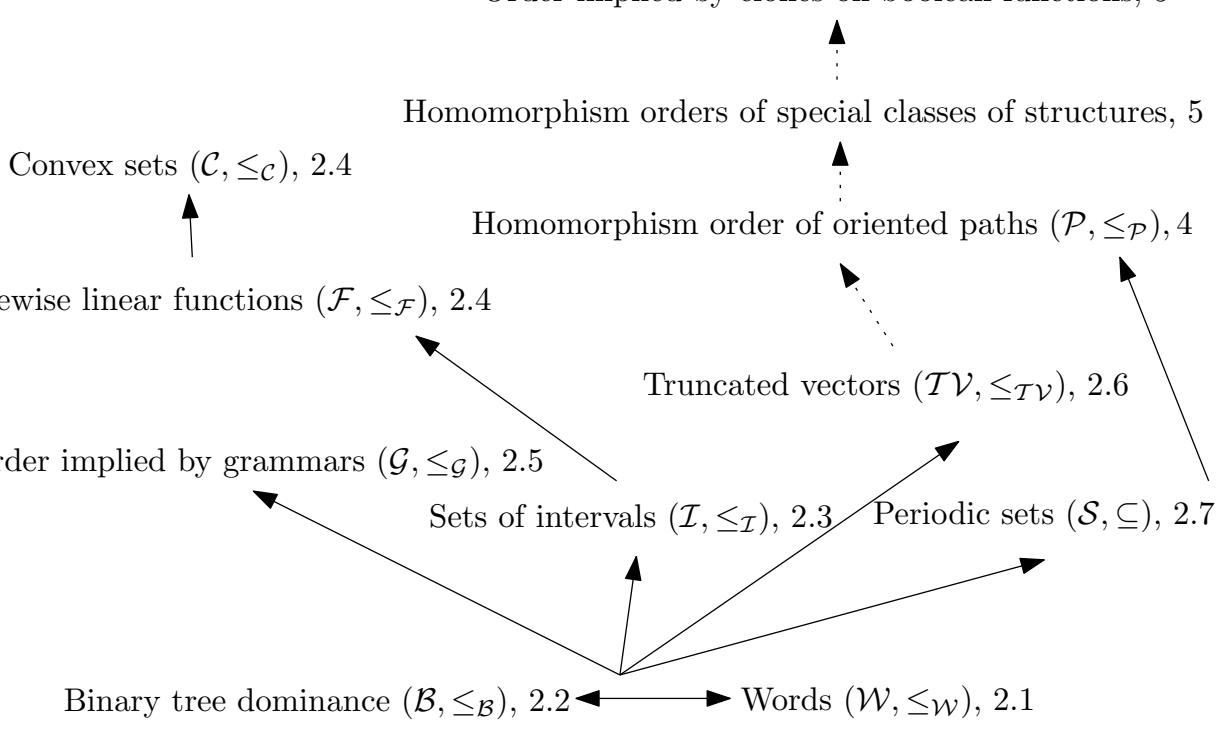

FIGURE 1. Embeddings presented in this paper

The class $\mathcal{K}$ of partial orders is on-line representable in the partial order $\left(P, \leq_{P}\right)$ if player $A$ has a winning strategy.

On-line representation (describing winning strategy of $A$ ) is a convenient way of showing the universality of given partial order. In particular it transforms problem of embedding countable structures into a finite problem of extending the existing partial embedding by next element.

We say that a partial order $\left(P, \leq_{P}\right)$ has the extension property if the following holds: for any finite mutually disjoint subsets $L, G, U \subseteq P$ there exist a vertex $v \in P$ such that $v^{\prime}<_{P} v$ for each $v^{\prime} \in L, v<_{P} v^{\prime}$ for each $v^{\prime} \in G$ and neither $v \leq_{P} v^{\prime}$ nor $v^{\prime} \leq_{P} v$ for each $v^{\prime} \in U$. The extension property is a stronger form of on-line representability of any partial order. Using a zig-zag argument it is easy to show that a partial order having the extension property is homogeneous (and thus generic).

In Section 3 we describe a finite representation of the generic partial order related to Conway's surreal numbers. Somewhat surprisingly, this is the only known finite presentation of the generic partial order [9]. The constructions of universal partial orders are easier, but they are often not generic. We discuss reasons why other structures fail to be ultrahomogeneous. In particular we will look for gaps in the partial order. Recall that the gap in a partial order $\left(P, \leq_{P}\right)$ is a pair of elements $v, v^{\prime} \in P$ such that $v<_{\mathcal{B}} v^{\prime}$. A partial order having no gaps is called dense. We will show examples of universal partial orders both with gaps and without gaps but still failing to be generic.

\section{Examples of Universal Partial Orders}

To prove the universality of a given partially ordered set is often a difficult task $[\mathbf{5}, \mathbf{2 0}, \mathbf{1 0}, \mathbf{1 6}]$. However, the individual proofs, even if developed independently, use similar tools. We demonstrate this by isolating a "master" construction (in 
Section 2.1). This construction is then embedded into partial orders defined by other structures (as listed above). We shall see that the representation of this particular order is flexible enough to simplify further embeddings.

2.1. Word representation. The set of all words over the alphabet $\Sigma=\{0,1\}$ is denoted by $\{0,1\}^{*}$. For words $W, W^{\prime}$ we write $W \leq_{w} W^{\prime}$ if and only if $W^{\prime}$ is an initial segment (left factor) of $W$. Thus we have, for example, $\{011000\} \leq_{w}\{011\}$ and $\{010111\} \not \leq w\{011\}$.

Definition 2.1. Denote by $\mathcal{W}$ the class of all finite subsets $A$ of $\{0,1\}^{*}$ such that no distinct words $W, W^{\prime}$ in $A$ satisfy $W \leq_{w} W^{\prime}$. For $A, B \in \mathcal{W}$ we put $A \leq_{\mathcal{W}} B$ when for each $W \in A$ there exists $W^{\prime} \in B$ such that $W \leq_{w} W^{\prime}$.

Obviously $\left(\mathcal{W}, \leq_{\mathcal{W}}\right)$ is a partial order (antisymmetry follows from the fact that $A$ is an antichain in the order $\leq_{w}$ ).

Definition 2.2. For a set $A$ of finite words denote by $\min A$ the set of all minimal words in $A$ (i.e. all $W \in A$ such that there is no $W^{\prime} \in A$ satisfying $\left.W^{\prime}<_{w} W\right)$.

Now we show that there is an on-line embedding of any finite partial order to $\left(\mathcal{W}, \leq_{\mathcal{W}}\right)$. Let $[n]$ be the set $\{1,2, \ldots, n\}$. The partial orders will be restricted to those whose vertex sets are sets $[n]$ (for some $n>1$ ) and the vertices will always be embedded in the natural order. Given a partial order $\left([n], \leq_{P}\right)$ let $\left([i], \leq_{P_{i}}\right)$ denote the partial order induced by $\left([n], \leq_{P}\right)$ on the set of vertices $[i]$.

Our main construction is the function $\Psi$ mapping partial orders $\left([n], \leq_{P}\right)$ to elements of $\left(\mathcal{W}, \leq_{\mathcal{W}}\right)$ defined as follows:

\section{DEFINITION 2.3 .}

Let $L\left([n], \leq_{P}\right)$ be the union of all $\Psi\left([m], \leq_{P_{m}}\right), m<n, m \leq_{P} n$.

Let $U\left([n], \leq_{P}\right)$ be the set of all words $W$ such that $W$ has length $n$, the last letter is 0 and for each $m<n, n \leq_{P} m$ there is a $W^{\prime} \in \Psi\left([m], \leq_{P_{m}}\right)$ such that $W$ is an initial segment of $W^{\prime}$.

Finally, let $\Psi\left([n], \leq_{P}\right)$ be $\min \left(L\left([n], \leq_{P}\right) \cup U\left([n], \leq_{P}\right)\right)$.

In particular, $L\left([1], \leq_{P}\right)=\emptyset, U\left([1], \leq_{P}\right)=\{0\}, \Psi\left([1], \leq_{P}\right)=\{0\}$.

The main result of this section is the following:

TheOREM 2.4. Given a partial order $\left([n], \leq_{P}\right)$ we have:

(1) For every $i, j \in[n]$,

$$
i \leq_{P} j \text { if and only if } \Psi\left([i], \leq_{P_{i}}\right) \leq_{\mathcal{W}} \Psi\left([j], \leq_{P_{j}}\right)
$$

and

$$
\Psi\left([i], \leq_{P_{i}}\right)=\Psi\left([j], \leq_{P_{j}}\right) \text { if and only if } i=j .
$$

(This says that the mapping $\Phi(i)=\Psi\left([i], \leq_{P_{i}}\right)$ is an embedding of $([n]$, $\left.\leq_{P}\right)$ into $\left.\left(\mathcal{W}, \leq_{\mathcal{W}}\right)\right)$,

(2) For every $S \subseteq[n]$ there is a word $W$ of length $n$ such that for each $k \leq n$, $\{W\} \leq_{\mathcal{W}} \Psi\left([k], \leq_{P_{k}}\right)$ if and only if either $k \in S$ or there is a $k^{\prime} \in S$ such that $k^{\prime} \leq_{P} k$.

The on-line embedding $\Phi$ is illustrated by the following example: 


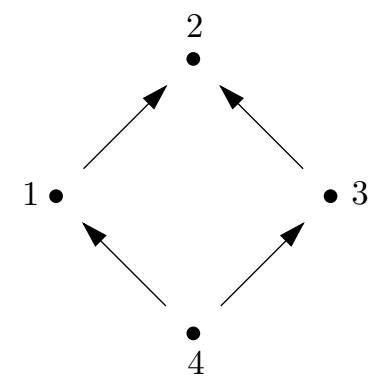

Figure 2. The partial order $\left([4], \leq_{P}\right)$.

EXAmPLE 2.5. The partial order $\left([4], \leq_{P}\right)$ depicted in Figure 2 has the following values of $\Psi\left([k], \leq_{P_{k}}\right), k=1,2,3,4$ :

$$
\begin{array}{lll}
L\left([1], \leq_{P_{1}}\right)=\emptyset, & U\left([1], \leq_{P_{1}}\right)=\{0\}, & \Psi\left([1], \leq_{P_{1}}\right)=\{0\}, \\
L\left([2], \leq_{P_{2}}\right)=\{0\}, & U\left([2], \leq_{P_{2}}\right)=\{00,10\}, & \Psi\left([2], \leq_{P_{2}}\right)=\{0,10\}, \\
L\left([3], \leq_{P_{3}}\right)=\emptyset, & U\left([3], \leq_{P_{3}}\right)=\{000,100\}, & \Psi\left([3], \leq_{P_{3}}\right)=\{000,100\}, \\
L\left([4], \leq_{P_{4}}\right)=\emptyset, & U\left([4], \leq_{P_{4}}\right)=\{0000\}, & \Psi\left([4], \leq_{P_{4}}\right)=\{0000\} .
\end{array}
$$

Proof (of Theorem 2.4). We proceed by induction on $n$.

The theorem obviously holds for $n=1$.

Now assume that the theorem holds for every partial order $\left([i], \leq_{P_{i}}\right), i=$ $1, \ldots, n-1$.

We first show that $(2)$ holds for $\left([n], \leq_{P}\right)$. Fix $S \subseteq\{1,2, \ldots, n\}$. Without loss of generality assume that for each $m \leq n$ such that there is an $m^{\prime} \in S$ with $m^{\prime} \leq_{P} m$, we also have $m \in S$ (i.e. $S$ is closed upwards). By the induction hypothesis, there is a word $W$ of length $n-1$ such that for each $n^{\prime}<n,\{W\} \leq_{\mathcal{W}} \Psi\left(\left[n^{\prime}\right], \leq_{P_{n^{\prime}}}\right)$ if and only if $n^{\prime} \in S$. Given the word $W$ we can construct a word $W^{\prime}$ of length $n$ such that $\left\{W^{\prime}\right\} \leq_{\mathcal{W}} \Psi\left(\left[n^{\prime}\right], \leq_{P_{n^{\prime}}}\right)$ if and only if $n^{\prime} \in S$. To see this, consider the following cases:

(1) $n \in S$

(a) $\{W\} \leq_{\mathcal{W}} \Psi\left([n], \leq_{P}\right)$. Put $W^{\prime}=W 0$. Since $\left\{W^{\prime}\right\} \leq_{\mathcal{W}}\{W\}, W^{\prime}$ obviously has the property.

(b) $\{W\} \not \mathbb{W}_{\mathcal{W}} \Psi\left([n], \leq_{P}\right)$. In this case we have $m \in S$ for each $m<$ $n, n \leq_{P} m$, and thus $\{W\} \leq_{\mathcal{W}} \Psi\left([m], \leq_{P_{m}}\right)$. By the definition of $\leq_{\mathcal{W}}$, for each such $m$ we have $W^{\prime \prime} \in \Psi\left([m], \leq_{P_{m}}\right)$ such that $W^{\prime \prime}$ is an initial segment of $W$. This implies that $W 0$ is in $U\left([n], \leq_{P}\right)$ and thus $\{W\} \leq_{\mathcal{W}} \Psi\left([n], \leq_{P}\right)$, a contradiction.

(2) $n \notin S$

(a) $\{W\} \not \mathbb{W}_{\mathcal{W}} \Psi\left([n], \leq_{P}\right)$. In this case we can put either $W^{\prime}=W 0$ or $W^{\prime}=W 1$

(b) $\{W\} \leq_{\mathcal{W}} \Psi\left([n], \leq_{P}\right)$. We have $\{W\} \leq_{\mathcal{W}} L\left([n], \leq_{P}\right)$-otherwise we would have $\{W\} \leq_{\mathcal{W}} \Psi\left([m], \leq_{P_{m}}\right) \leq_{\mathcal{W}} \Psi\left([n], \leq_{P}\right)$ for some $m<n$ and thus $n \in S$. Since $U\left([n], \leq_{P}\right)$ contains words of length $n$ whose last digit is 0 putting $W^{\prime}=W 1$ gives $\left\{W^{\prime}\right\} \not_{\mathcal{W}} U\left([n], \leq_{P}\right)$ and thus also $\left\{W^{\prime}\right\} \not{L} \mathcal{W} \Psi\left([m], \leq_{P_{m}}\right)$. 
This finishes the proof of property (2).

Now we prove (1). We only need to verify that for $m=1,2, \ldots, n-1$ we have $\Psi\left([n], \leq_{P}\right) \leq_{\mathcal{W}} \Psi\left([m], \leq_{P_{m}}\right)$ if and only if $n \leq_{P} m$ and $\Psi\left([m], \leq_{P_{m}}\right) \leq_{\mathcal{W}} \Psi\left([n], \leq_{P}\right)$ if and only if $m \leq{ }_{P} n$. The rest follows by induction. Fix $m$ and consider the following cases:

(1) $m \leq_{P} n$ implies $\Psi\left([m], \leq_{P_{m}}\right) \leq_{\mathcal{W}} \Psi\left([n], \leq_{P}\right)$ : This follows easily from the fact that every word in $\Psi\left([m], \leq_{P_{m}}\right)$ is in $L\left([n], \leq_{P}\right)$ and the initial segment of each word in $L\left([n], \leq_{P}\right)$ is in $\Psi\left([n], \leq_{P}\right)$.

(2) $n \leq_{P} m$ implies $\Psi\left([n], \leq_{P}\right) \leq_{\mathcal{W}} \Psi\left([m], \leq_{P_{m}}\right): U\left([n], \leq_{P}\right)$ is a maximal set of words of length $n$ with last digit 0 such that $U\left([n], \leq_{P}\right) \leq_{\mathcal{W}}$ $\Psi\left(\left[m^{\prime}\right], \leq_{P_{m^{\prime}}}\right)$ for each $m^{\prime}<n, n \leq_{P} m^{\prime}$, in particular for $m^{\prime}=m$. It suffices to show that $L\left([n], \leq_{P}\right) \leq_{\mathcal{W}} \Psi\left([m], \leq_{P_{m}}\right)$. For $W \in L\left([n], \leq_{P}\right)$, we have an $m^{\prime \prime}, m^{\prime \prime} \leq_{P} n \leq_{P} m$, such that $W \in \Psi\left(\left[m^{\prime \prime}\right], \leq_{P_{m^{\prime \prime}}}\right)$. From the induction hypothesis $\Psi\left(\left[m^{\prime \prime}\right], \leq_{P_{m^{\prime \prime}}}\right) \leq_{\mathcal{W}} \Psi\left([m], \leq_{P_{m}}\right)$-in particular the initial segment of $W$ is in $\Psi\left([m], \leq_{P_{m}}\right)$.

(3) $\Psi\left([m], \leq_{P_{m}}\right) \leq_{\mathcal{W}} \Psi\left([n], \leq_{P}\right)$ implies $m \leq_{P} n$ : Since $U\left([n], \leq_{P}\right)$ contains words longer than any word of $m$, we have $\Psi\left([m], \leq_{P_{m}}\right) \leq_{\mathcal{W}} L\left([n], \leq_{P}\right)$. By (2) for $S=\{m\}$ there is a word $W$ such that $\{W\} \leq_{\mathcal{W}} \Psi\left(\left[m^{\prime}\right], \leq_{P_{m^{\prime}}}\right)$ if and only if $m \leq_{P} m^{\prime}$. Since $\{W\} \leq_{\mathcal{W}} L\left([n], \leq_{P}\right)$, we have an $m^{\prime}$ such that $m \leq_{P} m^{\prime} \leq_{P} n$.

(4) $\Psi\left([n], \leq_{P}\right) \leq_{\mathcal{W}} \Psi\left([m], \leq_{P_{m}}\right)$ implies $n \leq_{P} m$ : We have $\Psi\left([n], \leq_{P}\right) \leq_{\mathcal{W}}$ $\Psi\left([m], \leq_{P_{m}}\right)$. By $(2)$ for $S=\{n\}$ there is a word $W$ such that $\{W\} \leq_{\mathcal{W}}$ $\Psi\left(\left[m^{\prime}\right], \leq_{P_{m^{\prime}}}\right)$ if and only if $n \leq_{P} m^{\prime}$. Since $\{W\} \leq_{\mathcal{W}} \Psi\left([m], \leq_{P_{m}}\right)$ we also have $n \leq_{P} m$.

Corollary 2.6. The partial order $(\mathcal{W}, \leq \mathcal{W})$ is universal.

Note that $\mathcal{W}$ fails to be a ultrahomogeneous partial order. For example the empty set is the minimal element. $\mathcal{W}$ is also not dense as shown by the following example:

$$
A=\{0\}, B=\{00,01\} .
$$

This is not unique gap - we shall characterize all gaps in $\left(\mathcal{W}, \leq_{\mathcal{W}}\right)$ after reformulating it in a more combinatorial setting in Section 2.2.

2.2. Dominance in the countable binary tree. As is well known, the Hasse diagram of the partial order $\left(\{0,1\}^{*}, \leq_{w}\right)$ forms a complete binary tree $T_{u}$ of infinite depth. Let $r$ be its root vertex (corresponding to the empty word). Using $T_{u}$ we can reformulate our universal partial order as:

Definition 2.7. The vertices of $\left(\mathcal{B}, \leq_{\mathcal{B}}\right)$ are finite sets $S$ of vertices of $T_{u}$ such that there is no vertex $v \in S$ on any path from $r$ to $v^{\prime} \in S$ except for $v^{\prime}$. (Thus $S$ is a finite antichain in the order of the tree $T$.)

We say that $S^{\prime} \leq_{\mathcal{B}} S$ if and only if for each path from $r$ to $v \in S$ there is a vertex $v^{\prime} \in S^{\prime}$.

Corollary 2.8. The partially ordered $\operatorname{set}\left(\mathcal{B}, \leq_{\mathcal{B}}\right)$ is universal.

Proof. $\left(\mathcal{B}, \leq_{\mathcal{B}}\right)$ is just a reformulation of $\left(\mathcal{W}, \leq_{\mathcal{W}}\right)$ and thus both partial orders are isomorphic. 


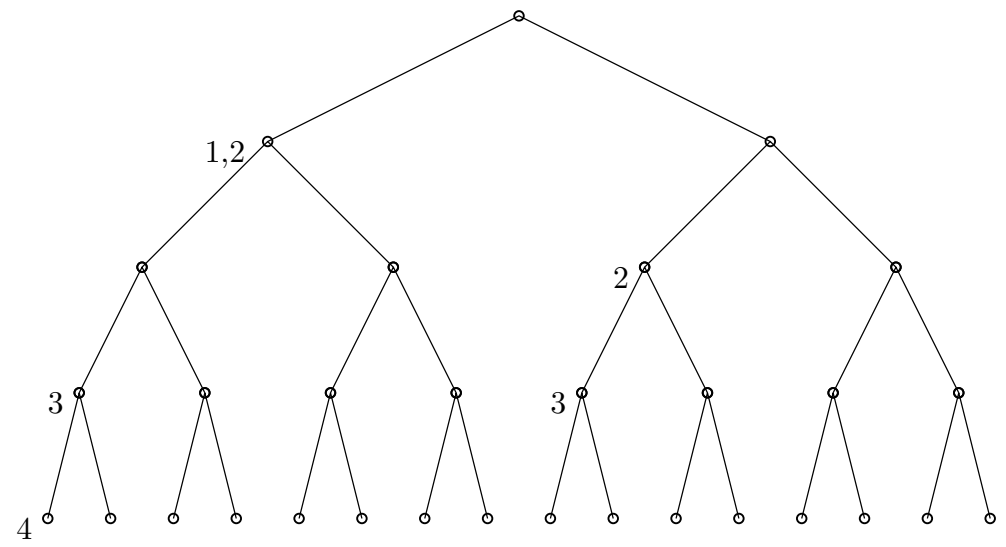

Figure 3. Tree representation of $\left([4], \leq_{P}\right)$ (Figure 2).

Figure 3 shows a portion of the tree $T$ representing the same partial order as in Figure 2.

The partial order $\left(\mathcal{B}, \leq_{\mathcal{B}}\right)$ offers perhaps a better intuitive understanding as to how the universal partial order is built from the very simple partial order $\left(\{0,1\}^{*}, \leq_{w}\right)$ by using sets of elements instead of single elements. Understanding this makes it easy to find an embedding of $\left(\mathcal{W}, \leq_{\mathcal{W}}\right)$ (or equivalently $\left(\mathcal{B}, \leq_{\mathcal{B}}\right)$ ) into a new structure by first looking for a way to represent the partial order $\left(\{0,1\}^{*}, \leq_{w}\right)$ within the new structure and then a way to represent subsets of $\{0,1\}^{*}$. This idea will be applied several times in the following sections.

Now we characterize gaps.

Proposition 2.9. $S<S^{\prime}$ is a gap in $\left(\mathcal{B}, \leq_{\mathcal{B}}\right)$ if and only if there exists an $s^{\prime} \in S^{\prime}$ such that

(1) there is a vertex $s \in S$ such that both sons $s_{0}, s_{1}$ of $s$ in the tree $T$ are in $S^{\prime}$

(2) $S \backslash\left\{s_{0}, s_{1}\right\}=S^{\prime} \backslash\{s\}$.

This means that all gaps in $\mathcal{B}$ result from replacing a member by its two sons.

Proof. Clearly any pair $S<S^{\prime}$ satisfying (1), (2) is a gap (as any $S \leq_{\mathcal{B}}$ $S^{\prime \prime} \leq_{\mathcal{B}} S^{\prime}$ has to contain $S^{\prime} \backslash\{s\}$, and either $s$ or the two vertices $\left.s_{0}, s_{1}\right)$.

Let $S \leq_{\mathcal{B}} S^{\prime}$ be a gap. If there are distinct vertices $s_{1}^{\prime}$ and $s_{2}^{\prime}$ in $S^{\prime}$ and $s_{1}, s_{2} \in S$ are such that $s_{i} \leq s_{i}^{\prime}$, i=1,2, then $S^{\prime \prime}$ defined as $\min \left(S \backslash\left\{s_{1}\right\}\right) \cup\left\{S_{1}^{\prime}\right\}$ satisfies $S<_{\mathcal{B}} S^{\prime \prime}<_{\mathcal{B}} S^{\prime}$.

Thus there is only one $S^{\prime} \in S^{\prime} \backslash S$ such that $s^{\prime}>s$ for an $s \in S$. However then there is only one such $s^{\prime}$ (so if $s_{1}, s_{2}$ are distinct then $S<S \backslash\left\{s_{2}\right\}<S^{\prime}$ ). Moreover it is either $s=s^{\prime} 0$ or $s=s^{\prime} 1$. Otherwise $S<S^{\prime}$ would not be a gap.

The abundance of gaps indicates that $\left(\mathcal{B}, \leq_{\mathcal{B}}\right)\left(\right.$ or $\left.\left(\mathcal{W}, \leq_{\mathcal{W}}\right)\right)$ are redundant universal partial orders. This makes them, in a way, far from being generic, since the generic partial order has no gaps. The next section has a variant of this partial order avoiding this problem. On the other hand gaps in partial orders are interesting and are related to dualities, see $[\mathbf{2 1}, \mathbf{1 9}]$. 
2.3. Intervals. We show that the vertices of $\left(\mathcal{W}, \leq_{\mathcal{W}}\right)$ can be coded by geometric objects ordered by inclusion. Since we consider only countable structures we restrict ourselves to objects formed from rational numbers.

While the interval on rationals ordered by inclusion can represent infinite increasing chains, decreasing chains or antichains, obviously this interval order has dimension 2 and thus fails to be universal. However considering multiple intervals overcomes this limitation:

Definition 2.10. The vertices of $\left(\mathcal{I}, \leq_{\mathcal{I}}\right)$ are finite sets $S$ of closed disjoint intervals $[a, b]$ where $a, b$ are rational numbers and $0 \leq a<b \leq 1$.

We put $A \leq_{\mathcal{I}} B$ when every interval in $A$ is covered by some interval of $B$.

In the other words elements of $\left(\mathcal{I}, \leq_{\mathcal{I}}\right)$ are finite sets of pairs of rational numbers. $A \leq_{\mathcal{I}} B$ holds if for every $[a, b] \in A$, there is an $\left[a^{\prime}, b^{\prime}\right] \in B$ such that $a^{\prime} \leq a$ and $b \leq b^{\prime}$.

Definition 2.11. A word $W=w_{1} w_{2} \ldots w_{t}$ on the alphabet $\{0,1\}$ can be considered as a number $0 \leq n_{W} \leq 1$ with ternary expansion:

$$
n_{W}=\sum_{i=1}^{t} w_{i} \frac{1}{3^{i}}
$$

For $A \in \mathcal{W}$, the representation of $A$ in $\mathcal{I}$ is then the following set of intervals:

$$
\Phi_{\mathcal{I}}^{\mathcal{W}}(A)=\left\{\left[n_{W}, n_{W}+\frac{2}{3^{|W|+1}}\right]: W \in A\right\} .
$$

The use of the ternary base might seem unnatural - indeed the binary base would suffice. The main obstacle to using the later is that the embedding of $\{00,01\}$ would be two intervals adjacent to each other overlapping in single point. One would need to take special care when taking the union of such intervals - we avoid this by using ternary numbers.

LEMMA 2.12. $\Phi_{\mathcal{I}}^{\mathcal{W}}$ is an embedding of $\left(\mathcal{W}, \leq_{\mathcal{W}}\right)$ into $\left(\mathcal{I}, \leq_{\mathcal{I}}\right)$.

Proof. It is sufficient to prove that for $W, W^{\prime}$ there is an interval $\left[n_{W}, n_{W}+\right.$ $\left.\frac{1}{\left.3^{|W|}\right]}\right]$ covered by an interval $\left[n_{W^{\prime}}, n_{W^{\prime}}+\frac{1}{3^{\left|W^{\prime}\right|}}\right]$ if and only if $W^{\prime}$ is initial segment of $W$. This follows easily from the fact that intervals represent precisely all numbers whose ternary expansion starts with $W$ with the exception of the upper bound itself. is:

ExAmple 2.13. The representation of $\left([4], \leq_{P}\right)$ as defined by Figure 2 in $\left(\mathcal{I}, \leq_{\mathcal{I}}\right)$

$$
\begin{aligned}
& \Phi_{\mathcal{I}}^{\mathcal{W}}\left(\Psi\left([1], \leq_{P_{1}}\right)\right)=\Phi_{\mathcal{I}}^{\mathcal{W}}(\{0\})=\left\{\left(0, \frac{2}{3^{2}}\right)\right\}, \\
& \Phi_{\mathcal{I}}^{\mathcal{W}}\left(\Psi\left([2], \leq_{P_{2}}\right)\right)=\Phi_{\mathcal{I}}^{\mathcal{W}}(\{0,10\})=\left\{\left(0, \frac{2}{3^{2}}\right),\left(\frac{1}{3}, \frac{1}{3}+\frac{2}{3^{3}}\right)\right\}, \\
& \Phi_{\mathcal{I}}^{\mathcal{W}}\left(\Psi\left([3], \leq_{P_{3}}\right)\right)=\Phi_{\mathcal{I}}^{\mathcal{W}}(\{000,100\})=\left\{\left(0, \frac{2}{3^{4}}\right),\left(\frac{1}{3}, \frac{1}{3}+\frac{2}{3^{4}}\right)\right\}, \\
& \Phi_{\mathcal{I}}^{\mathcal{W}}\left(\Psi\left([4], \leq_{P_{4}}\right)\right)=\Phi_{\mathcal{I}}^{\mathcal{W}}(\{0000\})=\left\{\left(0, \frac{2}{3^{5}}\right)\right\} .
\end{aligned}
$$

Corollary 2.14. The partial order $\left(\mathcal{I}, \leq_{\mathcal{I}}\right)$ is universal.

The partial order $\left(\mathcal{I}, \leq_{\mathcal{I}}\right)$ differs significantly from $\left(\mathcal{W}, \leq_{\mathcal{W}}\right)$ by the following:

Proposition 2.15. The partial order $\left(\mathcal{I}, \leq_{\mathcal{I}}\right)$ has no gaps (is dense). 
Proof. Take $A, B \in \mathcal{I}, A<_{\mathcal{I}} B$. Because all the intervals in both $A$ and $B$ are closed and disjoint, there must be at least one interval $I$ in $B$ that is not fully covered by intervals of $A$ (otherwise we would have $B \leq_{\mathcal{I}} A$ ). We may construct an element $C$ from $B$ by shortening the interval $I$ or splitting it into two disjoint intervals in a way such that $A<_{\mathcal{I}} C<_{\mathcal{I}} B$ holds.

Consequently the presence (and abundance) of gaps in most of the universal partial orders studied is not the main obstacle when looking for representations of partial orders. It is easy to see that $\left(\mathcal{I}, \leq_{\mathcal{I}}\right)$ is not generic.

By considering a variant of $\left(\mathcal{I}, \leq_{\mathcal{I}}\right)$ with open (instead of closed) intervals we obtain a universal partial order $\left(\mathcal{I}^{\prime}, \leq_{\mathcal{I}^{\prime}}\right)$ with gaps. The gaps are similar to the ones in $\left(\mathcal{B}, \leq_{\mathcal{B}}\right)$ created by replacing interval $(a, b)$ by two intervals $(a, c)$ and $(c, d)$. Half open intervals give a quasi-order containing a universal partial order.

2.4. Geometric representations. The representation as a set of intervals might be considered an artificially constructed structure. Partial orders represented by geometric objects are studied in [1]. It is shown that objects with $n$ "degrees of freedom" cannot represent all partial orders of dimension $n+1$. It follows that convex hulls used in the representation of the generic partial order cannot be defined by a constant number of vertices. We will show that even the simplest geometric objects with unlimited "degrees of freedom" can represent a universal partial order.

Definition 2.16. Denote by $\left(\mathcal{C}, \leq_{\mathcal{C}}\right)$ the partial order whose vertices are all convex hulls of finite sets of points in $\mathbb{Q}^{2}$, ordered by inclusion.

This time we will embed $\left(\mathcal{I}, \leq_{\mathcal{I}}\right)$ into $\left(\mathcal{C}, \leq_{\mathcal{C}}\right)$.

Definition 2.17. For every $A \in \mathcal{I}$ denote by $\Phi_{\mathcal{C}}^{\mathcal{I}}(A)$ the convex hull generated by the points:

$$
\left(a, a^{2}\right),\left(\frac{a+b}{2}, a b\right),\left(b, b^{2}\right), \text { for every }(a, b) \in A .
$$

See Figure 4 for the representation of the partial order in Figure 2.

Theorem 2.18. $\Phi_{\mathcal{C}}^{\mathcal{I}}$ is an embedding of $\left(\mathcal{I}, \leq_{\mathcal{I}}\right)$ to $\left(\mathcal{C}, \leq_{\mathcal{C}}\right)$.

Proof. All points of the form $\left(x, x^{2}\right)$ lie on a convex parabola $y=x^{2}$. The points $\left(\frac{a+b}{2}, a b\right)$ are the intersection of two tangents of this parabola at the points $\left(a, a^{2}\right)$ and $\left(b, b^{2}\right)$. Consequently all points in the construction of $\Phi_{\mathcal{C}}^{\mathcal{I}}(A)$ lie in a convex configuration.

We have $\left(x, x^{2}\right)$ in the convex hull $\Phi_{\mathcal{C}}^{\mathcal{I}}(A)$ if and only if there is $[a, b] \in A$ such that $a \leq x \leq b$. Thus for $A, B \in \mathcal{I}$ we have $\Phi_{\mathcal{C}}^{\mathcal{I}}(A) \leq_{\mathcal{C}} \Phi_{\mathcal{C}}^{\mathcal{I}}(B)$ implies $A \leq_{\mathcal{I}} B$.

To see the other implication, observe that the convex hull of $\left(a, a^{2}\right),\left(\frac{a+b}{2}, a b\right)$, $\left(b, b^{2}\right)$ is a subset of the convex hull of $\left(a^{\prime}, a^{\prime 2}\right),\left(\frac{a^{\prime}+b^{\prime}}{2}, a^{\prime} b^{\prime}\right),\left(b^{\prime}, b^{\prime 2}\right)$ for every $[a, b]$ that is a subinterval of $\left[a^{\prime}, b^{\prime}\right]$.

We have:

Corollary 2.19. The partial order $\left(\mathcal{C}, \leq_{\mathcal{C}}\right)$ is universal.

Remark 2.20. Our construction is related to Venn diagrams. Consider the partial order $\left([n], \leq_{P}\right)$. For the empty relation $\leq_{P}$ the representation constructed by $\Phi_{\mathcal{C}}^{\mathcal{I}}\left(\Phi_{\mathcal{I}}^{\mathcal{W}}(\Psi([n], \emptyset))\right)$ is a Venn diagram, by Theorem 2.4 (2). Statement 2 of Theorem 2.4 can be seen as a Venn diagram condition under the constraints imposed by $\leq_{P}$. 
1:

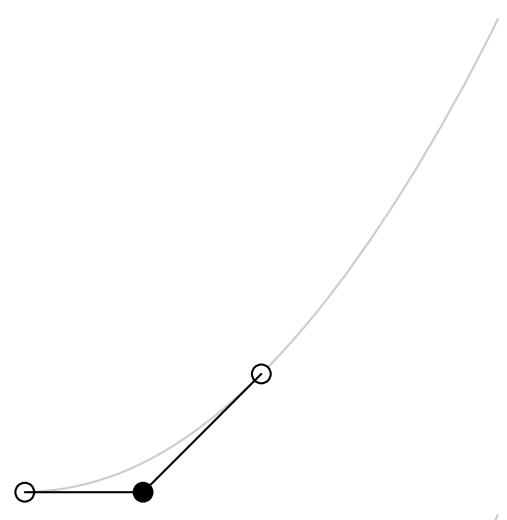

$3:$

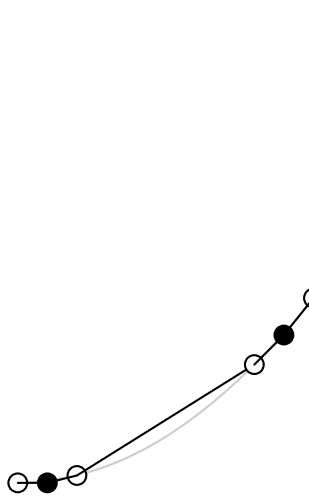

2:

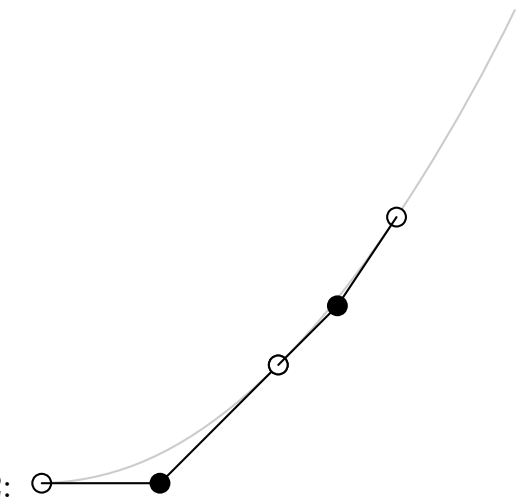

4:

Figure 4. Representation of the partial order $\left([4], \leq_{P}\right)$ in $\left(\mathcal{C}, \leq_{\mathcal{C}}\right)$.

The same construction can be applied to functions, and stated in a perhaps more precise manner.

Corollary 2.21. Consider the class $\mathcal{F}$ of all convex piecewise linear functions on the interval $(0,1)$ consisting of a finite set of segments, each with rational boundaries. Put $f \leq \mathcal{F} g$ if and only if $f(x) \leq g(x)$ for every $0 \leq x \leq 1$. Then the partial $\operatorname{order}\left(\mathcal{F}, \leq_{\mathcal{F}}\right)$ is universal.

Similarly the following holds:

TheOREM 2.22. Denote by $\mathcal{O}$ the class of all finite polynomials with rational coefficients. For $p, q \in \mathcal{O}$, put $p \leq_{\mathcal{O}} q$ if and only if $p(x) \leq q(x)$ for $x \in(0,1)$. The partial order $\left(\mathcal{O}, \leq_{\mathcal{O}}\right)$ is universal.

The proof of this theorem needs tools of mathematical analysis The proof of this theorem needs more involved tools of mathematical analysis and it will appear elsewhere (jointly with Robert Šámal).

2.5. Grammars. The rewriting rules used in a context-free grammar can be also used to define a universal partially ordered set. 
Definition 2.23. The vertices of $\left(\mathcal{G}, \leq_{\mathcal{G}}\right)$ are all words over the alphabet $\{\downarrow$, $\uparrow, 0,1\}$ created from the word 1 by the following rules:

$$
\begin{aligned}
& 1 \rightarrow \downarrow 11 \uparrow, \\
& 1 \rightarrow 0 .
\end{aligned}
$$

$W \leq_{\mathcal{G}} W^{\prime}$ if and only if $W$ can be constructed from $W^{\prime}$ by:

$$
\begin{aligned}
1 & \rightarrow \downarrow 11 \uparrow, \\
1 & \rightarrow 0, \\
\downarrow 00 \uparrow & \rightarrow 0 .
\end{aligned}
$$

$\left(\mathcal{G}, \leq_{\mathcal{G}}\right)$ is a quasi-order: the transitivity of $\leq_{\mathcal{G}}$ follows from the composition of lexical transformations.

Definition 2.24. Given $A \in \mathcal{W}$ construct $\Phi_{\mathcal{G}}^{\mathcal{W}}$ as follows:

(1) $\Phi_{\mathcal{C}}^{\mathcal{W}}(\emptyset)=0$.

(2) $\Phi_{\mathcal{G}}^{\mathcal{W}}(\{$ empty word $\})=1$.

(3) $\Phi_{\mathcal{G}}^{\mathcal{W}}(A)$ is defined as the concatenation $\downarrow \Phi_{\mathcal{G}}^{\mathcal{W}}\left(A_{0}\right) \Phi_{\mathcal{G}}^{\mathcal{W}}\left(A_{1}\right) \uparrow$, where $A_{0}$ is created from all words of $A$ starting with 0 with the first digit removed and $A_{1}$ is created from all words of $A$ starting with 1 with the first digit removed.

EXAMPLE 2.25. The representation of $\left([4], \leq_{P}\right)$ as defined by Figure 2 in $(\mathcal{G}$, $\leq_{\mathcal{G}}$ ) is as follows (see also the correspondence with the $\mathcal{B}$ representation in Figure 3 ):

$$
\begin{aligned}
& \Phi_{\mathcal{G}}^{\mathcal{W}}\left(\Psi\left([1], \leq_{P_{1}}\right)\right)=\Phi_{\mathcal{G}}^{\mathcal{W}}(\{0\})=\downarrow 10 \uparrow, \\
& \Phi_{\mathcal{G}}^{\mathcal{W}}\left(\Psi\left([2], \leq_{P_{2}}\right)\right)=\Phi_{\mathcal{G}}^{\mathcal{W}}(\{0,10\}) \quad=\downarrow 1 \downarrow 10 \uparrow \uparrow, \\
& \Phi_{\mathcal{C}}^{\mathcal{W}}\left(\Psi\left([3], \leq_{P_{3}}\right)\right)=\Phi_{\mathcal{C}}^{\mathcal{W}}(\{000,100\})=\downarrow \downarrow \downarrow 10 \uparrow 0 \uparrow \downarrow \downarrow 10 \uparrow 0 \uparrow \uparrow, \\
& \Phi_{\mathcal{G}}^{\mathcal{W}}\left(\Psi\left([4], \leq_{P_{4}}\right)\right)=\Phi_{\mathcal{G}}^{\mathcal{W}}(\{0000\})=\downarrow \downarrow \downarrow \downarrow 10 \uparrow 0 \uparrow 0 \uparrow 0 \uparrow .
\end{aligned}
$$

We state the following without proof as it follows straightforwardly from the definitions.

Proposition 2.26. For $A, B \in \mathcal{W}$ the inequality $A \leq_{\mathcal{W}} B$ holds if and only if $\Phi_{\mathcal{G}}^{\mathcal{W}}(A) \leq_{\mathcal{G}} \Phi_{\mathcal{G}}^{\mathcal{W}}(B)$.

$\left(\mathcal{G}, \leq_{\mathcal{G}}\right)$ is a quasi-order. We have:

Corollary 2.27. The quasi-order $\left(\mathcal{G}, \leq_{\mathcal{G}}\right)$ contains a universal partial order.

2.6. Multicuts and Truncated Vectors. A universal partially ordered structure similar to $(\mathcal{W}, \leq \mathcal{W})$, but less suitable for further embeddings, was studied in $[\mathbf{5}, \mathbf{1 6}, \mathbf{1 0}]$. While the structures defined in these papers are easily shown to be equivalent, their definition and motivations were different. [5] contains the first finite presentation of universal partial order. [16] first used the notion of on-line embeddings to (1) prove the universality of the structure and (2) as intermediate structure to prove the universality of the homomorphism order of multigraphs. The motivation for this structure came from the analogy with Dedekind cuts and thus its members were called multicuts. In [10] an essentially equivalent structure with the inequality reversed was used as an intermediate structure for the stronger result showing the universality of oriented paths. This time the structure arises in the context of orders of vectors (as the simple extension of the orders of finite dimension represented by finite vectors of rationals) resulting in name truncated vectors.

We follow the presentation in $[\mathbf{1 0}]$. 
Definition 2.28. Let $\vec{v}=\left(v_{1}, \ldots, v_{t}\right), \vec{v}^{\prime}=\left(v_{1}^{\prime}, \ldots, v_{t^{\prime}}^{\prime}\right)$ be $0-1$ vectors. We put:

$$
\vec{v} \leq_{\vec{v}} \vec{v}^{\prime} \text { if and only if } t \geq t^{\prime} \text { and } v_{i} \geq v_{i}^{\prime} \text { for } i=1, \ldots, t^{\prime} .
$$

Thus we have e.g. $(1,0,1,1,1)<_{\vec{v}}(1,0,0,1)$ and $(1,0,0,1)>_{\vec{v}}(1,1,1,1)$. An example of an infinite descending chain is e.g.

$$
(1)>_{\vec{v}}(1,1)>_{\vec{v}}(1,1,1)>_{\vec{v}} \ldots
$$

Any finite partially ordered set is representable by vectors with this ordering: for vectors of a fixed length we have just the reverse ordering of that used in the (Dushnik-Miller) dimension of partially ordered sets, see e.g. [21].

Definition 2.29. We denote by $\mathcal{T} V$ the class of all finite vector-sets. Let $\vec{V}$ and $\vec{V}^{\prime}$ be two finite sets of $0-1$ vectors. We put $\vec{V} \leq_{\mathcal{T} V} \vec{V}^{\prime}$ if and only if for every $\vec{v} \in \vec{V}$ there exists a $\vec{v}^{\prime} \in \vec{V}^{\prime}$ such that $\vec{v} \leq_{\vec{v}} \vec{v}^{\prime}$.

For a word $W$ on the alphabet $\{0,1\}$ we construct a vector $\vec{v}(W)$ of length $2|W|$ such that $2 n$-th element of vector $\vec{v}(W)$ is 0 if and only if the $n$-th character of $W$ is 0 , and the $(2 n+1)$-th element of the vector $\vec{v}(W)$ is 1 if and only if the $n$-th character of $W$ is 0 .

It is easy to see that $W \leq_{\mathcal{W}} W^{\prime}$ if and only if $\vec{v}(W) \leq_{\vec{v}} \vec{v}\left(W^{\prime}\right)$. The embedding $\Phi_{\mathcal{T} V}^{\mathcal{W}}:\left(\mathcal{W}, \leq_{\mathcal{W}}\right) \rightarrow\left(\mathcal{T} V, \leq_{\mathcal{T} V}\right)$ is constructed as follows:

$$
\Phi_{\mathcal{T} V}^{\mathcal{W}}=\{\vec{v}(W), W \in A\}
$$

For our example $\left([4], \leq_{P}\right)$ in Figure 2 we have embedding:

$$
\begin{aligned}
& \Phi_{\mathcal{T} V}^{\mathcal{W}}\left(\Psi\left([1], \leq_{P_{1}}\right)\right)=\Phi_{\mathcal{T} V}^{\mathcal{W}}(\{0\})=\{(0,1)\} \\
& \Phi_{\mathcal{T} V}^{\mathcal{W}}\left(\Psi\left([2], \leq_{P_{2}}\right)\right)=\Phi_{\mathcal{T} V}^{\mathcal{W}}(\{0,10\})=\{(0,1),(1,0,0,1)\}, \\
& \Phi_{\mathcal{T} V}^{\mathcal{W}}\left(\Psi\left([3], \leq_{P_{3}}\right)\right)=\Phi_{\mathcal{T} V}^{\mathcal{W}}(\{000,100\})=\{(0,1,0,1,0,1),(1,0,0,1,0,1)\}, \\
& \Phi_{\mathcal{T} V}^{\mathcal{W}}\left(\Psi\left([4], \leq_{P_{4}}\right)\right)=\Phi_{\mathcal{T} V}^{\mathcal{W}}(\{0000\})=\{(0,1,0,1,0,1,0,1)\} .
\end{aligned}
$$

COROllary 2.30. The quasi-order $\left(\mathcal{T} V, \leq_{\mathcal{T} V}\right)$ contains a universal partial order.

The structure $\left(\mathcal{T} V, \leq_{\mathcal{T} V}\right)$ as compared to $\left(\mathcal{W}, \leq_{\mathcal{W}}\right)$ is more complicated to use for further embeddings: the partial order of vectors is already a complex finiteuniversal partial order. The reason why the structure $(\mathcal{T} V, \leq \mathcal{T} V)$ was discovered first is is that it allows a remarkably simple on-line embedding that we outline now.

Again we restrict ourselves to the partial orders whose vertex sets are the sets $[n]$ (for some $n>1$ ) and we will always embed the vertices in the natural order. The function $\Psi^{\prime}$ mapping partial orders $\left([n], \leq_{P}\right)$ to elements of $\left(\mathcal{T} V, \leq_{\mathcal{T} V}\right)$ is defined as follows:

Definition 2.31. Let $\vec{v}\left([n], \leq_{P}\right)=\left(v_{1}, v_{2}, \ldots, v_{n}\right)$ where $v_{m}=1$ if and only if $n \leq_{\mathcal{P}} m, m \leq n$, otherwise $v_{m}=0$.

Let

$$
\Psi^{\prime}\left([n], \leq_{P}\right)=\left\{\vec{v}\left([m], \leq_{P_{m}}\right): m \in P, m \leq n, m \leq_{\mathcal{P}} n\right\} .
$$

For our example in Figure 2 we get a different (and more compact) embedding:

$$
\begin{array}{ll}
\vec{v}(1)=(1), & \Psi^{\prime}\left([1], \leq_{P_{1}}\right)=\{(1)\}, \\
\vec{v}(2)=(0,1), & \Psi^{\prime}\left([2], \leq_{P_{2}}\right)=\{(1),(0,1)\}, \\
\vec{v}(3)=(1,0,1), & \Psi^{\prime}\left([3], \leq_{P_{3}}\right)=\{(1,0,1)\}, \\
\vec{v}(4)=(1,1,1,1), & \Psi^{\prime}\left([4], \leq_{P}\right)=\{(1,1,1,1)\} .
\end{array}
$$


Theorem 2.32. Fix the partial order $\left([n], \leq_{P}\right)$. For every $i, j \in[n]$,

$$
i \leq_{P} j \text { if and only if } \Psi^{\prime}\left([i], \leq_{P_{i}}\right) \leq_{\mathcal{T} V} \Psi^{\prime}\left([j], \leq_{P_{j}}\right)
$$

and

$$
\Psi^{\prime}\left([i], \leq_{P_{i}}\right)=\Psi^{\prime}\left([j], \leq_{P_{j}}\right) \text { if and only if } i=j .
$$

(Or in the other words, the mapping $\Phi^{\prime}(i)=\Psi^{\prime}\left([i], \leq_{P_{i}}\right)$ is the embedding of $([n]$, $\left.\leq_{P}\right)$ into $\left(\mathcal{T} V, \leq_{\mathcal{T} V}\right)$ ).

The proof can be done via induction analogously as in the second part of the proof of Theorem 2.4. See our paper [10]. The main advantage of this embedding is that the size of the answer is $O\left(n^{2}\right)$ instead of $O\left(2^{n}\right)$.

2.7. Periodic sets. As the last finite presentation we mention the following what we believe to be very elegant description. Consider the partial order defined by inclusion on sets of integers. This partial order is uncountable and contains every countable partial order. We can however show the perhaps surprising fact that the subset of all periodic subsets (which has a very simple and finite description) is countably universal.

Definition 2.33. $S \subseteq \mathbb{Z}$ is $p$-periodic if for every $x \in S$ we have also $x+p \in S$ and $x-p \in S$.

For a periodic set $S$ with period $p$ denote by the signature $s(p, S)$ a word over the alphabet $\{0,1\}$ of length $p$ such that $n$-th letter is 1 if and only if $n \in S$.

By $\mathcal{S}$ we denote the class of all sets $S \subseteq \mathbb{Z}$ such that $S$ is $2^{n}$-periodic for some $n$.

Clearly every periodic set is determined by its signature and thus $(\mathcal{S}, \subseteq)$ is a finite presentation. We consider the ordering of periodic sets by inclusion and prove:

TheOREM 2.34. The partial order $(\mathcal{S}, \subseteq)$ is universal.

Proof. We embed $\left(\mathcal{W}, \leq_{\mathcal{W}}\right)$ into $(\mathcal{S}, \subseteq)$ as follows: For $A \in \mathcal{W}$ denote by $\Phi_{\mathcal{S}}^{\mathcal{W}}(A)$ the set of integers such that $n \in \Phi_{\mathcal{S}}^{\mathcal{W}}(A)$ if and only if there is $W \in A$ and the $|A|$ least significant digits of the binary expansion of $n$ forms a reversed word $W$ (when the binary expansion has fewer than $|W|$ digits, add 0 as needed).

It is easy to see that $\Phi_{S}^{\mathcal{W}}(A)$ is $2^{n}$-periodic, where $n$ is the length of longest word in $W$, and $\Phi_{\mathcal{S}}^{\mathcal{W}}(A) \subseteq \Phi_{\mathcal{S}}^{\mathcal{W}}\left(A^{\prime}\right)$ if and only if $A \leq_{\mathcal{W}} A^{\prime}$.

$(\mathcal{S}, \subseteq)$ is dense, but it fails to have the 3-extension property: there is no set strictly smaller than the set with signature 01 and greater than both sets with signatures 0100 and 0010 .

\section{Generic Poset and Conway numbers}

One of the striking (and concise) incarnations of the generic Rado graph is provided by the set theory: vertices of $\mathcal{R}$ are all sets in a fixed countable model $\mathfrak{M}$ of the theory of finite sets, and the edges correspond to pairs $\{A, B\}$ for which either $A \in B$ or $B \in A$. In [9] we aimed for a similarly concise representation of a generic partial order. That appeared to be a difficult task and we had to settle for the weaker notion of "finite presentation". At present $[\mathbf{9}]$ is the only finite presentation of the generic partial order. This is related to Conway surreal numbers $[\mathbf{1 2}, \mathbf{2}]$. 
In this section, for completeness we give the finite presentation of the generic partial order as shown in [9]. This construction is of independent interest as one can give a finite presentation of the rational Urysohn space along the same lines [7]. We work in a fixed countable model $\mathfrak{M}$ of the theory of finite sets extended by a single atomic set $\boldsymbol{\omega}$. To represent ordered pairs $\left(M_{L}, M_{R}\right)$, we use following notation:

$$
\begin{gathered}
M_{L}=\{A ; A \in M, \mathscr{\Phi} \notin A\} ; \\
M_{R}=\{A ;(A \cup\{\dot{\omega}\}) \in M, \mathscr{\omega} \notin A\} .
\end{gathered}
$$

Definition 3.1. Define the partially ordered set $\left(\mathcal{P}_{\epsilon}, \leq_{\epsilon}\right)$ as follows:

The elements of $\mathcal{P}_{\in}$ are all sets $M$ with the following properties:

(1) (correctness)

(a) $\sigma \notin M$,

(b) $M_{L} \cup M_{R} \subset \mathcal{P}_{\epsilon}$,

(c) $M_{L} \cap M_{R}=\emptyset$.

(2) (ordering property) $\left(\{A\} \cup A_{R}\right) \cap\left(\{B\} \cup B_{L}\right) \neq \emptyset$ for each $A \in M_{L}, B \in$ $M_{R}$,

(3) (left completeness) $A_{L} \subseteq M_{L}$ for each $A \in M_{L}$,

(4) (right completeness) $B_{R} \subseteq M_{R}$ for each $B \in M_{R}$.

The relation of $\mathcal{P}_{\in}$ is denoted by $\leq_{\epsilon}$ and it is defined as follows: We put $M<_{\in} N$ if

$$
\left(\{M\} \cup M_{R}\right) \cap\left(\{N\} \cup N_{L}\right) \neq \emptyset .
$$

We write $M \leq_{\in} N$ if either $M<_{\in} N$ or $M=N$.

The class $\mathcal{P}_{\in}$ is non-empty (as $M=\emptyset=(\emptyset \mid \emptyset) \in \mathcal{P}_{\in}$ ). (Obviously the correctness property holds. Since $M_{L}=\emptyset, M_{R}=\emptyset$, the ordering property and completeness properties follow trivially.)

Here are a few examples of non-empty elements of the structure $\mathcal{P}_{\in}$ :

$$
\begin{gathered}
(\emptyset \mid \emptyset), \\
(\emptyset \mid\{(\emptyset \mid \emptyset)\}), \\
(\{(\emptyset \mid \emptyset),(\emptyset \mid\{(\emptyset \mid \emptyset)\})\} \mid \emptyset) .
\end{gathered}
$$

It is a non-trivial fact that $\left(\mathcal{P}_{\epsilon}, \leq_{\epsilon}\right)$ is a partially ordered set. This will be proved after introducing some auxiliary notions:

Definition 3.2. Any element $W \in\left(A \cup A_{R}\right) \cap\left(B \cup B_{L}\right)$ is called a witness of the inequality $A<_{\in} B$.

Definition 3.3. The level of $A \in \mathcal{P}_{\in}$ is defined as follows:

$$
\begin{aligned}
l(\emptyset) & =0, \\
l(A) & =\max \left(l(B): B \in A_{L} \cup A_{R}\right)+1 \text { for } A \neq \emptyset .
\end{aligned}
$$

We observe the following facts (which follow directly from the definition of $\mathcal{P}_{\in}$ ):

FACT 3.4. $X<_{\in} A<_{\in} Y$ for every $A \in \mathcal{P}_{\in}, X \in A_{L}$ and $Y \in A_{R}$.

FACT 3.5. $A \leq_{\in} W^{A B} \leq_{\in} B$ for any $A<_{\in} B$ and witness $W^{A B}$ of $A<_{\in} B$.

FACT 3.6. Let $A<_{\in} B$ and let $W^{A B}$ be a witness of $A<_{\in} B$. Then $l\left(W^{A B}\right) \leq$ $\min (l(A), l(B))$, and either $l\left(W^{A B}\right)<l(A)$ or $l\left(W^{A B}\right)<l(B)$.

First we prove transitivity. 
Lemma 3.7. The relation $\leq_{\epsilon}$ is transitive on the class $\mathcal{P}_{\epsilon}$.

Proof. Assume that three elements $A, B, C$ of $\mathcal{P} \in$ satisfy $A<_{\epsilon} B<_{\epsilon} C$. We prove that $A<\in C$ holds. Let $W^{A B}$ and $W^{B C}$ be witnesses of the inequalities $A<\in B$ and $B<\in C$ respectively. First we prove that $W^{A B} \leq \in W^{B C}$. We distinguish four cases (depending on the definition of the witness):

(1) $W^{A B} \in B_{L}$ and $W^{B C} \in B_{R}$.

In this case it follows from Fact 3.4 that $W^{A B}<\in W^{B C}$.

(2) $W^{A B}=B$ and $W^{B C} \in B_{R}$.

Then $W^{B C}$ is a witness of the inequality $B<\in W^{B C}$ and thus $W^{A B}<\in W^{B C}$.

(3) $W^{A B} \in B_{L}$ and $W^{B C}=B$.

The inequality $W^{A B} \leq_{\in} W^{B C}$ follows analogously to the previous case.

(4) $W^{A B}=W^{B C}=B$ (and thus $W^{A B} \leq \in W^{B C}$ ).

In the last case $B$ is a witness of the inequality $A<_{\in} C$. Thus we may assume that $W^{A B} \neq \in W^{B C}$. Let $W^{A C}$ be a witness of the inequality $W^{A B}<_{\in} W^{B C}$. Finally we prove that $W^{A C}$ is a witness of the inequality $A<_{\epsilon} C$. We distinguish three possibilities:

(1) $W^{A C}=W^{A B}=A$.

(2) $W^{A C}=W^{A B}$ and $W^{A C} \in A_{R}$.

(3) $W^{A C} \in W_{R}^{A B}$, then also $W^{A C} \in A_{R}$ from the completeness property.

It follows that either $W^{A C}=A$ or $W^{A C} \in A_{R}$. Analogously either $W^{A C}=C$ or $W^{A C} \in C_{L}$ and thus $W^{A C}$ is the witness of inequality $A<_{\in} C$.

Lemma 3.8. The relation $<_{\epsilon}$ is strongly antisymmetric on the class $\mathcal{P}_{\epsilon}$.

Proof. Assume that $A<_{\epsilon} B<_{\epsilon} A$ is a counterexample with minimal $l(A)+$ $l(B)$. Let $W^{A B}$ be a witness of the inequality $A<_{\in} B$ and $W^{B A}$ a witness of the reverse inequality. From Fact 3.5 it follows that $A \leq_{\in} W^{A B} \leq_{\in} B \leq_{\in} W^{B A} \leq_{\epsilon}$ $A \leq_{\in} W^{A B}$. From the transitivity we know that $W^{A B} \leq_{\in} W^{B A}$ and $W^{B A} \leq_{\epsilon}$ $W^{A B}$.

Again we consider 4 possible cases:

(1) $W^{A B}=W^{B A}$.

From the disjointness of the sets $A_{L}$ and $A_{R}$ it follows that $W^{A B}=$ $W^{B A}=A$. Analogously we obtain $W^{A B}=W^{B A}=B$, which is a contradiction.

(2) Either $W^{A B}=A$ and $W^{B A}=B$ or $W^{A B}=B$ and $W^{B A}=A$.

Then a contradiction follows in both cases from the fact that $l(A)<$ $l(B)$ and $l(B)<l(A)$ (by Fact 3.6).

(3) $W^{A B} \neq A, W^{A B} \neq B, W^{A B} \neq W^{B A}$.

Then $l\left(W^{A B}\right)<l(A)$ and $l\left(W^{A B}\right)<l(B)$. Additionally we have $l\left(W^{B A}\right) \leq l(A)$ and $l\left(W^{B A}\right) \leq l(B)$ and thus $A$ and $B$ is not a minimal counter example.

(4) $W^{B A} \neq A, W^{B A} \neq B, W^{A B} \neq W^{B A}$.

The contradiction follows symmetrically to the previous case from the minimality of $l(A)+l(B)$.

Theorem 3.9. $\left(\mathcal{P}_{\epsilon}, \leq_{\epsilon}\right)$ is a partially ordered set. 
Proof. Reflexivity of the relation follow directly from the definition, transitivity and antisymmetry follow from Lemmas 3.7 and 3.8.

Now we are ready to prove the main result of this section:

THEOREM 3.10. $\left(\mathcal{P}_{\in}, \leq_{\epsilon}\right)$ is the generic partially ordered set for the class of all countable partial orders.

First we show the following lemma:

Lemma 3.11. $\left(\mathcal{P}_{\epsilon}, \leq_{\epsilon}\right)$ has the extension property.

Proof. Let $M$ be a finite subset of the elements of $\mathcal{P}_{\in}$. We want to extend the partially ordered set induced by $M$ by the new element $X$. This extension can be described by three subsets of $M: M_{-}$containing elements smaller than $X, M_{+}$ containing elements greater than $X$, and $M_{0}$ containing elements incomparable with $X$. Since the extended relation is a partial order we have the following properties of these sets:

I. Any element of $M_{-}$is strictly smaller than any element of $M_{+}$,

II. $B \leq_{\in} A$ for no $A \in M_{-}, B \in M_{0}$,

III. $A \leq_{\in} B$ for no $A \in M_{+}, B \in M_{0}$,

IV. $M_{-}, M_{+}$and $M_{0}$ form a partition of $M$.

Put

$$
\begin{aligned}
& \overline{M_{-}}=\bigcup_{B \in M_{-}} B_{L} \cup M_{-}, \\
& \overline{M_{+}}=\bigcup_{B \in M_{+}} B_{R} \cup M_{+} .
\end{aligned}
$$

We verify that the properties I., II., III., IV. still hold for sets $\overline{M_{-}}, \overline{M_{+}}, M_{0}$.

ad I. We prove that any element of $\overline{M_{-}}$is strictly smaller than any element of $\overline{M_{+}}$:

Let $A \in \overline{M_{-}}, A^{\prime} \in \overline{M_{+}}$. We prove $A<_{\in} A^{\prime}$. By the definition of $\overline{M_{-}}$ there exists $B \in M_{-}$such that either $A=B$ or $A \in B_{L}$. By the definition of $\overline{M_{+}}$there exists $B^{\prime} \in M_{+}$such that either $A^{\prime}=B^{\prime}$ or $A^{\prime} \in B_{R}^{\prime}$. By the definition of $<_{\epsilon}$ we have $A \leq_{\epsilon} B, B<_{\epsilon} B^{\prime}$ (by I.) and $B^{\prime} \leq_{\epsilon} A^{\prime}$ again by the definition of $<_{\epsilon}$. It follows $A<_{\epsilon} A^{\prime}$.

ad II. We prove that $B \leq_{\in} A$ for no $A \in \overline{M_{-}}, B \in M_{0}$ :

Let $A \in \overline{M_{-}}, B \in M_{0}$ and let $A^{\prime} \in M_{-}$satisfy either $A=A^{\prime}$ or $A \in A_{L}^{\prime}$. We know that $B \not \leq \in A^{\prime}$ and as $A \leq_{\in} A^{\prime}$ we have also $B \not \leq \in A$.

ad III. To prove that $A \leq_{\in} B$ for no $A \in \overline{M_{+}}, B \in M_{0}$ we can proceed similarly to ad II.

ad IV. We prove that $\overline{M_{-}}, \overline{M_{+}}$and $M_{0}$ are pairwise disjoint:

$\overline{M_{-}} \cap \overline{M_{+}}=\emptyset$ follows from I. $\overline{M_{-}} \cap M_{0}=\emptyset$ follows from II. $\overline{M_{+}} \cap M_{0}=$ $\emptyset$ follows from III.

It follows that $A=\left(\overline{M_{-}} \mid \overline{M_{+}}\right)$is an element of $\mathcal{P}_{\in}$ with the desired inequalities for the elements in the sets $M_{-}$and $M_{+}$.

Obviously each element of $M_{-}$is smaller than $A$ and each element of $M_{+}$is greater than $A$.

It remains to be shown that each $N \in M_{0}$ is incomparable with $A$. However we run into a problem here: it is possible that $A=N$. We can avoid this problem 
by first considering the set:

$$
M^{\prime}=\bigcup_{B \in M} B_{R} \cup M
$$

It is then easy to show that $B=\left(\emptyset \mid M^{\prime}\right)$ is an element of $\mathcal{P}_{\in}$ strictly smaller than all elements of $M$.

Finally we construct the set $A^{\prime}=\left(A_{L} \cup\{B\} \mid A_{R}\right)$. The set $A^{\prime}$ has the same properties with respect to the elements of the sets $M_{-}$and $M_{+}$and differs from any set in $M_{0}$. It remains to be shown that $A^{\prime}$ is incomparable with $N$.

For contrary, assume for example, that $N<\in A^{\prime}$ and $W^{N A^{\prime}}$ is the witness of the inequality. Then $W^{N A^{\prime}} \in \overline{M_{-}}$and $N \leq_{\in} W^{N A^{\prime}}$. Recall that $N \in M_{0}$. From IV. above and the definition of $A^{\prime}$ it follows that $N<\in W^{N A^{\prime}}$. From ad III. above it follows that there is no choice of elements with $N<\in W^{N A^{\prime}}$, a contradiction.

The case $N>\in A^{\prime}$ is analogous.

Proof. Proof of Theorem 3.10 follows by combining Lemma 3.11 and fact that extension property imply both universality and ultrahomogeneity of the partial order.

Example 3.12. Consider partial order $\left(P, \leq_{P}\right)$ depicted in Figure 2. The function $c$ embedding $\left(P, \leq_{P}\right)$ to $\left(\mathcal{P}_{\in}, \leq_{\mathcal{P}_{\epsilon}}\right)$ can be defined as:

$$
\begin{aligned}
& c(1)=(\emptyset \mid \emptyset), \\
& c(2)=(\emptyset \mid\{(\emptyset \mid \emptyset)\}), \\
& c(3)=(\{(\emptyset \mid \emptyset),(\emptyset \mid\{(\emptyset \mid \emptyset)\})\} \mid \emptyset), \\
& c(4)=(\{(\emptyset \mid\{(\emptyset \mid \emptyset)\})\} \mid\{(\{(\emptyset \mid \emptyset),(\emptyset \mid\{(\emptyset \mid \emptyset)\})\} \mid \emptyset)\}) .
\end{aligned}
$$

3.1. Remark on Conway's surreal numbers. Recall the definition of surreal numbers, see $[\mathbf{1 2}]$. (For a recent generalization see $[\mathbf{3}]$ ). Surreal numbers are defined recursively together with their linear order. We briefly indicate how the partial order $\left(\mathcal{P}_{\in}, \leq_{\mathcal{P} \in}\right)$ fits into this scheme.

Definition 3.13. A surreal number is a pair $x=\left\{x^{L} \mid x^{R}\right\}$, where every member of the sets $x^{L}$ and $x^{R}$ is a surreal number and every member of $x^{L}$ is strictly smaller than every member of $x^{R}$.

We say that a surreal number $x$ is less than or equal to the surreal number $y$ if and only if $y$ is not less than or equal to any member of $x^{L}$ and any member of $y^{R}$ is not less than or equal to $x$.

We will denote the class of surreal numbers by $\mathbb{S}$.

$\mathcal{P}_{\in}$ may be thought of as a subset of $\mathbb{S}$ (we recursively add $\boldsymbol{\Phi}$ to express pairs $\left.x^{L}, x^{R}\right)$. The recursive definition of $A \in \mathcal{P}_{\in}$ leads to the following order which we define explicitly:

Definition 3.14. For elements $A, B \in \mathcal{P}_{\in}$ we write $A \leq_{\mathbb{S}} B$, when there is no $l \in A_{L}$ such that $B \leq_{\mathbb{S}} l$ and no $r \in B_{R}$ such that $r \leq_{\mathbb{S}} A$.

$\leq_{\mathbb{S}}$ is a linear order of $\mathcal{P}_{\in}$ and it is the restriction of Conway's order. It is in fact a linear extension of the partial order $\left(\mathcal{P}_{\epsilon}, \leq_{\epsilon}\right)$ :

Theorem 3.15. For any $A, B \in \mathcal{P}_{\in}, A<_{\in} B$ implies $A<_{\mathbb{S}} B$. 
Proof. We proceed by induction on $l(A)+l(B)$.

For empty $A$ and $B$ the theorem holds as they are not comparable by $<_{\epsilon}$.

Let $A<\in B$ with $W^{A B}$ as a witness. If $W^{A B} \neq A, B$, then $A<_{\mathbb{S}} W^{A B}<\mathbb{S} B$ by induction. In the case $A \in B_{L}$, then $A<_{\mathbb{S}} B$ from the definition of $<_{\mathbb{S}}$.

\section{Universality of Graph Homomorphisms}

Perhaps the most natural order between finite models is induced by homomorphisms. The universality of the homomorphism order for the class of all finite graphs was first shown by $[\mathbf{2 0}]$.

Numerous other classes followed (see e. g. [20]) but planar graphs (and other topologically restricted classes) presented a problem.

The homomorphism order on the class of finite paths was studied in [19]. It has been proved it is a dense partial order (with the exception of a few gaps which were characterized; these gaps are formed by all core-path of height $\leq 4)$. [19] also rises (seemingly too ambitious) question whether it is a universal partial order. This has been resolved in $[\mathbf{8}, \mathbf{1 0}]$ by showing that finite oriented paths with homomorphism order are universal. In this section we give a new proof of this result. The proof is simpler and yields a stronger result (see Theorem 4.11).

Recall that an oriented path $P$ of length $n$ is any oriented graph $(V, E)$ where $V=\left\{v_{0}, v_{1}, \ldots, v_{n}\right\}$ and for every $i=1,2, \ldots, n$ either $\left(v_{i-1}, v_{i}\right) \in E$ or $\left(v_{i}, v_{i-1}\right) \in$ $E$ (but not both), and there are no other edges. Thus an oriented path is any orientation of an undirected path.

Denote by $\left(\mathcal{P}, \leq_{\mathcal{P}}\right)$ the class of all finite paths ordered by homomorphism order. Given paths $P=(V, E), P^{\prime}=\left(V^{\prime}, E^{\prime}\right)$, a homomorphism is a mapping $\varphi: V \rightarrow V^{\prime}$ which preserves edges:

$$
(x, y) \in E \Longrightarrow(\varphi(x), \varphi(y)) \in E^{\prime} .
$$

For paths $P$ and $P^{\prime}$ we write $P \leq_{\mathcal{P}} P^{\prime}$ if and only if there is homomorphism $\varphi: P \rightarrow P^{\prime}$

To show the universality of oriented paths, we will construct an embedding of $(\mathcal{S}, \subseteq)$ to $\left(\mathcal{P}, \leq_{\mathcal{P}}\right)$. Recall that the class $\mathcal{S}$ denotes the class of all periodic subsets of $\mathbb{Z}$ (see Section 2.7). This is a new feature, which gives a new, more streamlined and shorter proof of the $[\mathbf{8}]$. The main difference of the proof in $[\mathbf{8}, \mathbf{1 0}]$ and the one presented here is the use of $(\mathcal{S}, \subseteq)$ as the base of the representation instead of $(\mathcal{T} V, \leq \mathcal{T} V)$. The linear nature of graph homomorphisms among oriented paths make it very difficult to adapt many-to-one mapping involved in $\leq_{\mathcal{T} V}$. The cyclic mappings of $(\mathcal{S}, \subseteq)$ are easier to use.

Let us introduce terms and notations that are useful when speaking of homomorphisms between paths. (We follow standard notations as e.g. in $[\mathbf{6}, \mathbf{1 9}]$.)

While oriented paths do not make a difference between initial and terminal vertices, we will always consider paths in a specific order of vertices from the initial to the terminal vertex. We denote the initial vertex $v_{0}$ and the terminal vertex $v_{n}$ of $P$ by $i n(P)$ and $\operatorname{term}(P)$ respectively. For a path $P$ we will denote by $\overleftarrow{P}$ the flipped path $P$ with order of vertices $v_{n}, v_{n-1}, \ldots, v_{0}$. For paths $P$ and $P^{\prime}$ we denote by $P P^{\prime}$ the path created by the concatenation of $P$ and $P^{\prime}$ (i.e. the disjoint union of $P$ and $P^{\prime}$ with $\operatorname{term}(P)$ identified with $\left.\operatorname{in}\left(P^{\prime}\right)\right)$.

The length of a path $P$ is the number of edges in $P$. The algebraic length of a path $P$ is the number of forwarding minus the number of backwarding edges in 


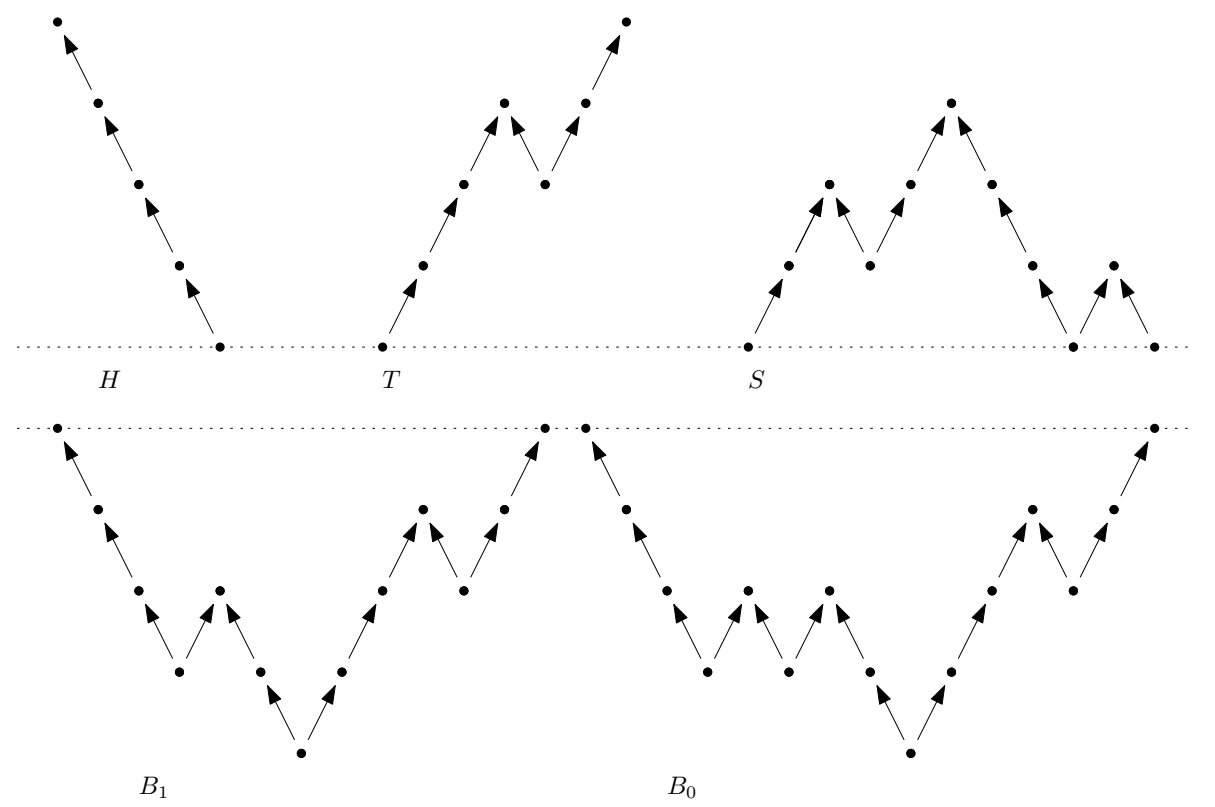

Figure 5. Building blocks of $p(W)$.

$P$. Thus the algebraic length of a path could be negative. The level $l_{P}\left(v_{i}\right)$ of $v_{i}$ is the algebraic length of the subpath $\left(p_{0}, p_{1}, \ldots, p_{i}\right)$ of $P$. The distance between vertices $p_{i}$ and $p_{j}, d_{P}\left(p_{i}, p_{j}\right)$, is given by $|j-i|$. The algebraic distance, $a_{P}\left(p_{i}, p_{j}\right)$, is $l_{P}\left(v_{j}\right)-l_{P}\left(v_{i}\right)$.

Denote by $\varphi: P \rightarrow P^{\prime}$ a homomorphism from path $P$ to $P^{\prime}$. Observe that we always have $d_{P}\left(p_{i}, p_{j}\right) \leq d_{P^{\prime}}\left(\varphi\left(p_{i}\right), \varphi\left(p_{j}\right)\right)$ and $a_{P}\left(p_{i}, p_{j}\right)=a_{P^{\prime}}\left(\varphi\left(p_{i}\right), \varphi\left(p_{j}\right)\right)$. We will construct paths in such a way that every homomorphism $\varphi$ between path $P$ and $P^{\prime}$ must map the initial vertex of $P$ to the initial vertex of $P^{\prime}$ and thus preserve levels of vertices (see Lemma 4.4 bellow).

The basic building blocks if our construction are the paths shown in Figure 5 ( $H$ stands for head, $T$ for tail, $B$ for body and $S$ for šipka - arrow in Czech language). Their initial vertices appear on the left, terminal vertices on the right. Except for $H$ and $T$ the paths are balanced (i.e. their algebraic length is 0 ). We will construct paths by concatenating copies of these blocks. $H$ will always be the first path, $T$ always the last. (The dotted line in Figure 5 and Figure 6 determines vertices with level -4.)

Definition 4.1. Given a word $W$ on the alphabet $\{0,1\}$ of length $2^{n}$, we assign path $p(W)$ recursively as follows:

(1) $p(0)=B_{0}$.

(2) $p(1)=B_{1}$.

(3) $p(W)=p\left(W_{1}\right) S \overleftarrow{p\left(W_{2}\right)}$ where $W_{1}$ and $W_{2}$ are words of length $2^{n-1}$ such that $W=W_{1} W_{2}$.

Put $\bar{p}(W)=H p(W) T$. 


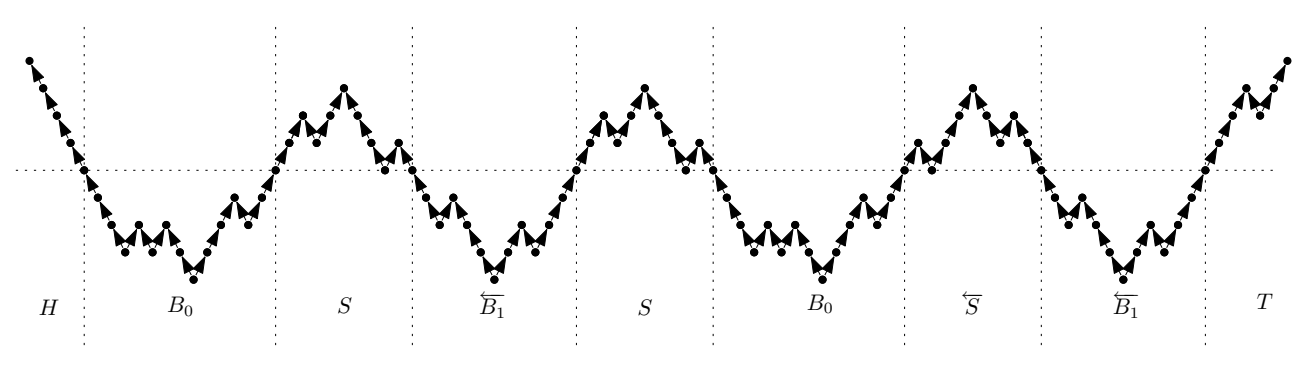

FiguRE 6. $\bar{p}(0110)$.

EXAMPLE 4.2. For a periodic set $S, s(4, S)=0110$, we construct $\bar{p}(s(4, S))$ in the following way:

$$
\begin{gathered}
p(0)=B_{0} \\
p(1)=B_{1} \\
p(01)=B_{0} S \overleftarrow{B_{1}} \\
p(10)=B_{1} S \overleftarrow{B_{0}} \\
p(0110)=B_{0} S \overleftarrow{B_{1}} S B_{0} \overleftarrow{S} \overleftarrow{B_{1}} \\
\bar{p}(0110)=H B_{0} S \overleftarrow{B_{1}} S B_{0} \overleftarrow{S} \overleftarrow{B_{1}} T
\end{gathered}
$$

See Figure 6.

The key result of our construction is given by the following:

Proposition 4.3. Fix a periodic set $S$ of period $2^{k}$ and a periodic set $S^{\prime}$ of period $2^{k^{\prime}}$. There is a homomorphism

$$
\varphi: \bar{p}\left(s\left(2^{k}, S\right)\right) \rightarrow \bar{p}\left(s\left(2^{k^{\prime}}, S^{\prime}\right)\right)
$$

if and only if $S \subseteq S^{\prime}$ and $k^{\prime} \leq k$.

If a homomorphism $\varphi$ exists, then $\varphi$ maps the initial vertex of $\bar{p}\left(s\left(2^{k}, S\right)\right)$ to the initial vertex of $\bar{p}\left(s\left(2^{k^{\prime}}, S^{\prime}\right)\right)$. If $k^{\prime}=k$ then $\varphi$ maps the terminal vertex of $\bar{p}\left(s\left(2^{k}, S\right)\right)$ to the terminal vertex of $\bar{p}\left(s\left(2^{k^{\prime}}, S^{\prime}\right)\right)$. If $k^{\prime}<k$ then $\varphi$ maps the terminal vertex of $\bar{p}\left(s\left(2^{k}, S\right)\right)$ to the initial vertex of $\bar{p}\left(s\left(2^{k^{\prime}}, S^{\prime}\right)\right)$.

Prior to the proof of Proposition 4.3 we start with observations about homomorphisms between our special paths.

Lemma 4.4. Any homomorphism $\varphi: \bar{p}(W) \rightarrow \bar{p}\left(W^{\prime}\right)$ must map the initial vertex of $\bar{p}(W)$ to the initial vertex of $\bar{p}\left(W^{\prime}\right)$.

Proof. $\bar{p}(W)$ starts with the monotone path of 7 edges. The homomorphism $\varphi$ must map this path to a monotone path in $\bar{p}\left(W^{\prime}\right)$. The only such subpath of $\bar{p}\left(W^{\prime}\right)$ is formed by first 8 vertices of $\bar{p}\left(W^{\prime}\right)$.

It is easy to see that $\varphi$ cannot flip the path: If $\varphi$ maps the initial vertex of $\bar{p}(W)$ to the 8 th vertex of $\bar{p}\left(W^{\prime}\right)$ then $\bar{p}(W)$ has vertices at level -8 and because homomorphisms must preserve algebraic distances, they must map to the vertex of level 1 in $\bar{p}\left(W^{\prime}\right)$ and there is no such vertex in $\bar{p}\left(W^{\prime}\right)$. 
LeMma 4.5. Fix words $W, W^{\prime}$ of the same length $2^{k}$. Let $\varphi$ be a homomorphism $\varphi: p(W) \rightarrow p\left(W^{\prime}\right)$. Then $\varphi$ maps the initial vertex of $p(W)$ to the initial vertex of $p\left(W^{\prime}\right)$ if and only if $\varphi$ maps the terminal vertex of $p(W)$ to the terminal vertex of $p\left(W^{\prime}\right)$.

Proof. We proceed by induction on length of $W$ :

For $W=i$ and $W^{\prime}=j, i, j \in\{0,1\}$ we have $p(W)=B_{i}$ and $p\left(W^{\prime}\right)=B_{j}$. There is no homomorphism $B_{1} \rightarrow B_{0}$. The unique homomorphism $B_{0} \rightarrow B_{1}$ has the desired properties. The only homomorphism $B_{0} \rightarrow B_{0}$ is the isomorphism $B_{0} \rightarrow B_{0}$.

In the induction step put $W=W_{0} W_{1}$ and $W^{\prime}=W_{0}^{\prime} W_{1}^{\prime}$ where $W_{0}, W_{1}$, $W_{0}^{\prime}, W_{1}^{\prime}$ are words of length $2^{k-1}$. We have $p(W)=p\left(W_{0}\right) S \stackrel{S\left(W_{1}\right)}{\text { and }} p\left(W^{\prime}\right)=$

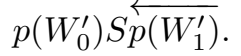

First assume that $\varphi$ maps $i n(p(W))$ to $i n\left(p\left(W^{\prime}\right)\right)$. Then $\varphi$ clearly maps $p\left(W_{0}\right)$ to $p\left(W_{0}^{\prime}\right)$ and thus by the induction hypothesis $\varphi$ maps $\operatorname{term}\left(p\left(W_{0}\right)\right)$ to $\operatorname{term}\left(p\left(W_{0}^{\prime}\right)\right)$. Because the vertices of $S$ are at different levels than the vertices of the final blocks $B_{0}$ or $B_{1}$ of $p\left(W_{0}^{\prime}\right)$, a copy of $S$ that follows in $p(W)$ after $p\left(W_{0}\right)$ must map to a copy of $S$ that follows in $p\left(W^{\prime}\right)$ after $p\left(W_{0}^{\prime}\right)$. Further $\varphi$ cannot flip $S$ and thus $\varphi$ maps $\operatorname{term}(S)$ to $\operatorname{term}(S)$. By same argument $\varphi$ maps $p\left(W_{1}\right)$ to $p\left(W_{1}^{\prime}\right)$. The initial vertex of $p\left(W_{1}\right)$ is the terminal vertex of $p(W)$ and it must map to the initial vertex of $p\left(W_{1}^{\prime}\right)$ and thus also the terminal vertex of $p\left(W^{\prime}\right)$.

The second possibility is that $\varphi$ maps $\operatorname{term}(p(W))$ to $\operatorname{term}\left(p\left(W^{\prime}\right)\right)$. This can be handled similarly (starting from the terminal vertex of paths in the reverse order).

Lemma 4.6. Fix periodic sets $S, S^{\prime}$ of the same period $2^{k}$. There is a homomorphism

$$
\varphi: p\left(s\left(2^{k}, S\right)\right) \rightarrow p\left(s\left(2^{k}, S^{\prime}\right)\right)
$$

mapping in $\left(p\left(s\left(2^{k}, S\right)\right)\right)$ to $\operatorname{in}\left(p\left(s\left(2^{k}, S^{\prime}\right)\right)\right)$ if and only if $S \subseteq S^{\prime}$.

Proof. If $S \subseteq S^{\prime}$ then the Lemma follows from the construction of $p\left(s\left(2^{k}, S\right)\right)$. Every digit 1 of $s\left(2^{k}, S\right)$ has a corresponding copy of $B_{1}$ in $p\left(s\left(2^{k}, S\right)\right)$ and every digit 0 has a corresponding copy of $B_{0}$ in $p\left(s\left(2^{k}, S\right)\right)$. It is easy to build a homomorphism $\varphi$ by concatenating a homomorphism $B_{0} \rightarrow B_{1}$ and identical maps of $S, B_{0}$ and $B_{1}$.

In the opposite direction, assume existence of homomorphism $\varphi$ from $p\left(s\left(2^{k}, S\right)\right)$ to $p\left(s\left(2^{k}, S^{\prime}\right)\right)$. By the assumption and Lemma $4.5, \varphi$ must be map $\operatorname{term}\left(p\left(s\left(2^{k}, S\right)\right)\right)$ to $\operatorname{term}\left(p\left(s\left(2^{k}, S^{\prime}\right)\right)\right)$. Because $S$ use vertices at different levels than $B_{0}$ and $B_{1}$, all copies of $S$ must be mapped to copies of $S$. Similarly copies of $B_{0}$ and $B_{1}$ must be mapped to copies of $B_{0}$ or $B_{1}$. If $S \nsubseteq S^{\prime}$ then there is position $i$ such that $i$-th letter of $s\left(2^{k}, S\right)$ is 1 and $i$-th letter of $s\left(2^{k}, S^{\prime}\right)$ is 0 . It follows that the copy of $B_{1}$ corresponding to this letter would have to map to a copy of $B_{0}$. This contradicts with the fact that there is no homomorphism $B_{1} \rightarrow B_{0}$.

Lemma 4.7 (folding). For a word $W$ of length $2^{k}$, there is a homomorphism

$$
\varphi: \bar{p}(W W) \rightarrow \bar{p}(W)
$$

mapping in $(\bar{p}(W W))$ to in $(\bar{p}(W))$ and term $(\bar{p}(W W))$ to in $(\bar{p}(W))$. 
Proof. By definition

$$
\bar{p}(W W)=H p(W) S \overleftarrow{p(W)} T
$$

and

$$
\bar{p}(W)=H p(W) T .
$$

The homomorphism $\varphi$ maps the first copy of $p(W)$ in $\bar{p}(W W)$ to a copy of $p(W)$ in $\bar{p}(W)$, a copy of $S$ is mapped to $T$ such that the terminal vertex of $S$ maps to the initial vertex of $T$ and thus it is possible to map a copy of $\overleftarrow{p(W)}$ in $\bar{p}(W W)$ to the same copy of $p(W)$ in $\bar{p}(W)$.

We will use the folding Lemma iteratively. By composition of homomorphisms there is also homomorphism $p(W W W W) \rightarrow p(W W) \rightarrow p(W)$. (From the path constructed from $2^{k}$ copies of $W$ to $p(W)$.)

Proof (of Proposition 4.3). Assume the existence of a homomorphism $\varphi$ as in Proposition 4.3. First observe that $k^{\prime} \leq k$ (if $k<k^{\prime}$ then there is a copy of $T$ in $\bar{p}\left(s\left(2^{k}, S\right)\right)$ would have to map into the middle of $\bar{p}\left(s\left(2^{k^{\prime}}, S^{\prime}\right)\right)$, but there are no vertices at the level 0 in $\bar{p}\left(s\left(2^{k^{\prime}}, S^{\prime}\right)\right)$ except for the initial and terminal vertex).

For $k=k^{\prime}$ the statement follows directly from Lemma 4.6.

For $k^{\prime}<k$ denote by $W^{\prime \prime}$ the word that consist of $2^{k-k^{\prime}}$ concatenations of $W^{\prime}$. Consider a homomorphism $\varphi^{\prime}$ from $p(W)$ to $p\left(W^{\prime \prime}\right)$ mapping $i n(p(W))$ to in $\left(p\left(W^{\prime \prime}\right)\right)$. $W$ and $W^{\prime \prime}$ have the same length and such a homomorphism exists by Lemma 4.6 if and only if $S \subseteq S^{\prime}$. Applying Lemma 4.7 there is a homomorphism $\varphi^{\prime \prime}: p\left(W^{\prime \prime}\right) \rightarrow p\left(W^{\prime}\right)$. A homomorphism $\varphi$ can be obtained by composing $\varphi^{\prime}$ and $\varphi^{\prime \prime}$. It is easy to see that any homomorphism $\bar{p}(W) \rightarrow \bar{p}\left(W^{\prime}\right)$ must follow the same scheme of "folding" the longer path $\bar{p}(W)$ into $\bar{p}\left(W^{\prime}\right)$ and thus there is a homomorphism $\varphi$ if and only if $S \subseteq S^{\prime}$. We omit the details.

For a periodic set $S$ denote by $S^{(i)}$ the inclusion maximal periodic subset of $S$ with period $i$. (For example for $s(4, S)=0111$ we have $s\left(2, S^{(2)}\right)=01$.)

Definition 4.8. For $S \in \mathcal{S}$ let $i$ be the minimal integer such that $S$ has period $2^{i}$. Let $\Phi_{\mathcal{P}}^{\mathcal{S}}(S)$ be the concatenation of the paths

$$
\begin{gathered}
H, \\
\bar{p}\left(s\left(1, S^{(1)}\right)\right) \overleftarrow{\bar{p}\left(s\left(1, S^{(1)}\right)\right)}, \\
\bar{p}\left(s\left(2, S^{(2)}\right)\right) \overleftarrow{\bar{p}\left(s\left(2, S^{(2)}\right)\right)}, \\
\bar{p}\left(s\left(4, S^{(4)}\right)\right) \overleftarrow{\bar{p}\left(s\left(4, S^{(4)}\right)\right)}, \\
\ldots, \\
\bar{p}\left(s\left(2^{i-1}, S^{\left(2^{i-1}\right)}\right)\right) \overleftarrow{\bar{p}\left(s\left(2^{i-1}, S^{\left(2^{i-1}\right)}\right)\right)}, \\
\bar{p}\left(s\left(2^{i}, S\right)\right) \overleftarrow{\bar{p}\left(s\left(2^{i}, S\right)\right)} .
\end{gathered}
$$

TheOREM 4.9. $\Phi_{\mathcal{P}}^{\mathcal{S}}(v)$ is an embedding of $(\mathcal{S}, \subseteq)$ to $\left(\mathcal{P}, \leq_{\mathcal{P}}\right)$. 
Proof. Fix $S$ and $S^{\prime}$ in $\mathcal{S}$ of periods $2^{i}$ and $2^{i^{\prime}}$ respectively.

Assume that $S \subseteq S^{\prime}, i>i^{\prime}$. Then the homomorphism $\varphi: \Phi_{\mathcal{P}}^{\mathcal{S}}(S) \rightarrow \Phi_{\mathcal{P}}^{\mathcal{S}}\left(S^{\prime}\right)$ can be constructed via the concatenation of homomorphisms:

$$
\begin{aligned}
& H \rightarrow H, \\
& \bar{p}\left(s\left(1, S^{(1)}\right)\right) \overleftarrow{\bar{p}\left(s\left(1, S^{(1)}\right)\right)} \rightarrow \bar{p}\left(s\left(1, S^{(1)}\right)\right) \overleftarrow{\bar{p}\left(s\left(1, S^{(1)}\right)\right)} \\
& \bar{p}\left(s\left(1, S^{(2)}\right)\right) \overleftarrow{\bar{p}\left(s\left(1, S^{(2)}\right)\right)} \rightarrow \bar{p}\left(s\left(2, S^{(2)}\right)\right) \overleftarrow{\bar{p}\left(s\left(2, S^{(2)}\right)\right)} \\
& \text {... } \\
& \bar{p}\left(s\left(2^{i^{\prime}-1}, S^{\left(2^{i^{\prime}-1}\right)}\right)\right) \overleftarrow{\bar{p}\left(s\left(2^{i^{\prime}-1}, S^{\left(2^{i^{\prime}-1}\right)}\right)\right)} \rightarrow \bar{p}\left(s\left(2^{i^{\prime}-1}, S^{\prime\left(2^{i^{\prime}-1}\right)}\right)\right) \bar{p}\left(s\left(2^{i^{\prime}-1}, S^{\prime\left(2^{i^{\prime}-1}\right)}\right)\right) \\
& \bar{p}\left(s\left(2^{i^{\prime}}, S^{\left(2^{i^{\prime}}\right)}\right)\right) \overleftarrow{\bar{p}\left(s\left(2^{i^{\prime}}, S^{\left(2^{i^{\prime}}\right)}\right)\right)} \rightarrow \bar{p}\left(s\left(2^{i^{\prime}}, S^{\prime}\right)\right) \\
& \bar{p}\left(s\left(2^{i^{\prime}+1}, S^{\left(2^{i^{\prime}+1}\right)}\right)\right) \overleftarrow{p\left(s\left(2^{i^{\prime}+1}, S^{\left(2^{i^{\prime}+1}\right)}\right)\right)} \rightarrow \bar{p}\left(s\left(2^{i^{\prime}}, S^{\prime}\right)\right) \\
& \bar{p}\left(s\left(2^{i}, S\right)\right) \overleftarrow{\bar{p}\left(s\left(2^{i^{\prime}}, S\right)\right)} \rightarrow \bar{p}\left(s\left(2^{i^{\prime}}, S^{\prime}\right)\right) .
\end{aligned}
$$

Individual homomorphisms exists by Proposition 4.3. For $i \leq i^{\prime}$ the construction is even easier.

In the opposite direction assume that there is a homomorphism $\varphi: \Phi_{\mathcal{P}}^{\mathcal{S}}(S) \rightarrow$ $\Phi_{\mathcal{P}}^{\mathcal{S}}\left(S^{\prime}\right) . \Phi_{\mathcal{P}}^{\mathcal{S}}(S)$ starts by two concatenations of $H$ and thus a long monotone path and using a same argument as in Lemma $4.4, \varphi$ must map the initial vertex of $\Phi_{\mathcal{P}}^{\mathcal{S}}(S)$ to the initial vertex of $\Phi_{\mathcal{P}}^{\mathcal{S}}\left(S^{\prime}\right)$. It follows that $\varphi$ preserves levels of vertices. It follows that for every $k=1,2,4, \ldots, 2^{i}, \varphi \operatorname{must} \operatorname{map} \bar{p}\left(s\left(k, S^{(k)}\right)\right)$ to $\bar{p}\left(s\left(k^{\prime}, S^{\prime\left(k^{\prime}\right)}\right)\right)$ for some $k^{\prime} \leq k, k^{\prime}=1,2,4, \ldots, 2^{i^{\prime}}$. By application of Proposition 4.3 it follows that $S^{(k)} \subseteq S^{\prime\left(k^{\prime}\right)}$. In particular $S \subseteq S^{\prime\left(k^{\prime}\right)}$. This holds only if $S \subseteq S^{\prime}$.

THEOREM $4.10([\mathbf{8}])$. The quasi order $\left(\mathcal{P}, \leq_{\mathcal{P}}\right)$ contains universal partial order.

In fact our new proof of Corollary 4.10 gives the following strengthening for rooted homomorphisms of paths. A plank $(P, r)$ is an oriented path rooted at the initial vertex $r=i n(P)$. Given planks $(P, r)$ and $\left(P^{\prime}, r^{\prime}\right)$, a homomorphism $\varphi:(P, r) \rightarrow\left(P^{\prime}, r^{\prime}\right)$ is a homomorphism $P \rightarrow P^{\prime}$ such that $\varphi(r)=r^{\prime}$.

THEOREM 4.11. The quasi order formed by all planks ordered by the existence of homomorphisms contains a universal partial order.

\section{Related results}

The universality of oriented paths implies the universality of the homomorphism order of many naturally defined classes of structures (such as undirected planar or series-parallel graphs) ordered by homomorphism via the indicator construction (see [10], [18]). By similar techniques the universality of homomorphism the order on labelled partial orders is shown in [13].

Lehtonen and Nešetřil [14] consider also the partial order defined on boolean functions in the following way. Each clone $\mathcal{C}$ on a fixed base set $A$ determines a quasiorder on the set of all operations on $A$ by the following rule: $f$ is a $\mathcal{C}$-minor of $g$ if $f$ can be obtained by substituting operations from $\mathcal{C}$ for the variables of $g$. Using embedding homomorphism order on hypergraphs, it can be shown that a clone $\mathcal{C}$ on $\{0,1\}$ has the property that the corresponding $\mathcal{C}$ minor partial order is 
universal if and only if $\mathcal{C}$ is one of the countably many clones of clique functions or the clone of self-dual monotone functions (using the classification of Post classes).

It seems that in most cases the homomorphism order of classes of relational structures is either universal or fails to be universal for very simple reasons (such as the absence of infinite chains or anti-chains). [18] look for minimal minor closed classes of graphs that are dense and universal. They show that $\left(\mathcal{P}, \leq_{\mathcal{P}}\right)$ is a unique minimal class of oriented graphs which is both universal and dense. Moreover, they show a dichotomy result for any minor closed class $\mathcal{K}$ of directed trees. $\mathcal{K}$ is either universal or it is well-quasi-ordered. Situation seems more difficult for the case of undirected graphs, where such minimal classes are not known and only partial result on series-parallel graphs was obtained.

\section{Acknowledgement}

We thank Andrew Goodall and Robert Šámal for help and several remarks which improved the quality of this paper. We thank to the anonymous referee for an excellent job.

\section{References}

1. N. Alon, E. Scheinerman: Degrees of freedom versus dimension for containment orders, Order 5 (1988) 11-16. 2.

2. J. H. Conway: On numbers and games, London Math. Soc. Monographs, Academic press, 1976.

3. P. Ehrlich: Number Systems with Simplicity Hiearchies. A generalization of Conway's Theory of Surreal Numbers, J. of Symbolic Logic 66, 3 (2001), 1231-1258.

4. R. Fraïssé: Théorie des relations, North Holland, 1986.

5. Z. Hedrlín: On universal partly oriented sets and classes, J. Algebra 11 (1969), 503-509.

6. P. Hell, N. Nešetřil: Graphs and Homomorphisms, Oxford University Press, Oxford, 2004.

7. J. Hubička J. Nešetřil: A Finite Presentation of the rational Urysohn Space, Topology and Applications 155 (14) (2008), 1483-1492.

8. J. Hubička, J. Nešetřil: Finite Paths are Universal, Order 21 (2004), 181-200.

9. J. Hubička, J. Nešetřil: Finite presentation of homogeneous graphs, posets and Ramsey classes, Israel J. Math 149 (2005), 21-44.

10. J. Hubička, J. Nešetřil: On universal posets represented by means of trees and other simple graphs, European J. Comb. 26 (2005), 765-778.

11. A. S. Kechris, V. G. Pestov, S. Todorcevic: Fraïssé Limits, Ramsey Theory, and Topological Dynamics of Automorphism Groups, to appear in GAFA. Geom. Funct. Anal., 15 (2005), 106-189.

12. D. E. Knuth: Surreal Numbers, Addison Wesley, 1974.

13. E. Lehtonen: Labeled posets are universal, European J. Comb., 29 (2) (2008), 493-506.

14. E. Lehtonen, J. Nešetřil, Minors of Boolean functions with respect to clique functions and hypergraph homomorphisms, European J. Comb. 31(2010) 1981-1995.

15. J. Nešetřil: For graphs there are only four types of hereditary Ramsey Classes, J. Comb. Th. B, 46(2), (1989), 127-132.

16. J. Nešetrril: On Universality of Set Systems. KAM-DIMATIA Series 491, Charles University, 2000.

17. J. Nešetřil: Ramsey Theory. In: Handbook of Combinatorics (ed. R. L. Graham, M. Grötschel, L. Lovász), Elsevier, 1995, 1331-1403.

18. J. Nešetřil, Y. Nigussie, Minimal universal and dense minor closed classes, European Journal of Combin. 27 (2006), 1159-1171.

19. J. Nešetřil, X. Zhu, Path Homomorphisms. Math. Proc. Comb. Phil. Soc. (1996), 207-220.

20. A. Pultr, V. Trnková, Combinatorial, Algebraic and Topological Representations of Groups, Semigroups and Categories, North Holland, 1980.

21. W. T. Trotter: Dimension Theory John Hopkins Univ. Press, 1992. 
Department of Applied Mathematics and Institute of Theoretical Computer sciences (ITI), Charles University, Malostranské nám. 25, 11800 Praha 1, Czech Republic

E-mail address: hubicka@kam.mff.cuni.cz

Department of Applied Mathematics and Institute of Theoretical Computer sciences (ITI), Charles University, Malostranské nám. 25, 11800 Praha 1, Czech Republic

E-mail address: nesetril@kam.mff.cuni.cz 